\title{
X-ray Diffraction, Calorimetric, and Dielectric Relaxation Study of the Amorphous and Smectic States of a Main Chain Liquid Crystalline Polymer
}

\author{
Mario Encinar, ${ }^{* \dagger, \|, \perp}$ Aránzazu Martínez-Gómez, ${ }^{*,+, \|}$ Ramón G. Rubio, ${ }^{\dagger}$ Ernesto Pérez, ${ }^{\ddagger}$ Antonio Bello, ${ }^{\ddagger}$ \\ and Margarita G. Prolongo ${ }^{\S}$ \\ †Departamento de Química Física I, Facultad de Química, Universidad Complutense, 28040-Madrid, Spain
${ }_{\ddagger}^{\dagger}$ Instituto de Ciencia y Tecnología de Polímeros, ICTP-CSIC, Juan de la Cierva 3, 28006-Madrid, Spain
${ }^{\S}$ Departamento de Materiales y Producción Aeroespacial, ETSI Aeronáuticos, Universidad Politécnica, 28040-Madrid, Spain
}

ABSTRACT: Liquid crystalline polymers (LCPs) are complex systems that include features of both orientationally ordered mesophases and amorphous polymers. Frequently, the isotropic amorphous state cannot be studied due to the rapid mesophase formation. Here, a new main chain LCP, poly(triethyleneglycol methyl $p, p^{\prime}$ bibenzoate), PTEMeB, has been synthesized. It shows a rather slow mesophase formation making possible to study independently both the amorphous and the liquid crystalline states. The structure and phase transitions of PTEMeB have been investigated by calorimetry, variable-temperature MAXS/WAXS employing synchrotron radiation, and $\mathrm{X}$-ray diffraction in oriented fibers. These experiments have pointed out the presence of two glass transitions, related to the amorphous or to the liquid crystal phases. Additionally, the mesophase seems to be a coexistence of orthogonal and

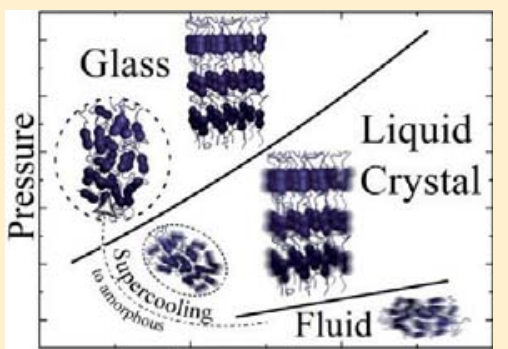

Temperature tilted smectic phases. A dielectric relaxation study of PTEMeB over broad ranges of temperature and pressure has been performed. The dynamic glass transition turns out to be slower for the amorphous state than for the liquid crystal. Monitoring of the $\alpha$ relaxation has allowed us to follow the isothermal mesophase formation at atmospheric pressure. Additionally, the dynamical behavior at high pressures has pointed out the fast formation of the mesophase induced by sudden pressure changes.

\section{INTRODUCTION}

A broad variety of favorable properties (including mechanical properties, dimensional stability, low gas permeability, etc.) and the possibility to tune them by relatively simple chemical variations in the structure make liquid crystal polymers (LCP) very attractive both from the industrial and academic points of view. These macromolecules can be considered as alternate copolymers formed by a rigid mesogenic group and a flexible one, usually called spacer. The complex behavior of the LCPs arises from the competition between the tendency of the mesogenic group to align and the polymeric tendency to maximize the entropy. Indeed, LCPs combine the characteristics of the liquid crystalline state and those of high molecular weight systems. ${ }^{1-3}$ Even though LCPs show anisotropy like conventional three-dimensional crystals and an extensive polymorphism, they lack 3D long-range positional order and are able to flow under stress. Some of the properties of the LCPs are their high mechanical stability, relatively low viscosity, long relaxation times, and a high molecular alignment, which enhance their processability by conventional techniques (extrusion, injection, and spinning). For instance, the inherent anisotropy of their chains has found one of the most interesting applications in the production of high modulus fibers. ${ }^{1,2}$

Another remarkable feature of LCPs is the possibility of freezing the orientational order of the mesophase just by cooling the sample at temperatures lower than the glass transition, $T_{\mathrm{g}}$, which is an attractive way to obtain anisotropic glasses with unique optical, mechanical, or electrical properties. ${ }^{4}$ Besides the technological interest, there are also fundamental aspects from the solid-state physics point of view. The analysis of the polymeric behavior in these systems must take into account special effects deriving from the presence of orientational order, and therefore, they can show special characteristics differing from those found in classical amorphous polymers. In the case of the glass transition, for instance, the activation of the segmental motion at temperatures above the $T_{\mathrm{g}}$ must be strongly related to the rotational freedom of the mesogens, which at the same time has to be compatible with the symmetry of the mesophase.

In principle, differences in the properties of the amorphous and liquid crystalline glasses are to be expected. A proper study of these differences requires the study of systems where both the pure isotropic amorphous and liquid-crystalline phases can be obtained. Unfortunately, it is usually rather difficult to quench the isotropic melt to an amorphous glass when liquid crystal formation is possible, and extraordinary quenching

Received: June 15, 2012

Revised: July 25, 2012

Published: July 26, 2012 
techniques may be needed. ${ }^{5}$ For this reason, only a few studies ${ }^{6-8}$ have been published on this subject. One of the main conclusions from these investigations is the existence of two different glass transitions for the liquid crystalline and amorphous states, the former laying at lower temperatures than the latter, as suggested from the corresponding calorimetric, dielectric, and dynamic-mechanical spectroscopy measurements.

The molecular dynamics of LCPs (with the mesogens either in the main chain, MCLCPs, or as side chains, SCLCPs) has attracted the attention of researchers for many years. ${ }^{9-11}$ For instance, in the case of SCLCPs, four different relaxation processes have been described. Zentel et al. found that the $\beta$ relaxation corresponded to the librational motion of the mesogenic group around the long molecular axis, while the $\gamma$ relaxation was associated to rotational fluctuations of the terminal groups linked to the mesogens. The $\alpha$ relaxation was assigned to the segmental dynamics of the main chain. A further relaxation, $\delta$, slower than the $\alpha$ one, corresponded to the tumbling motion as in nonpolymeric LCs. ${ }^{12-14}$

The $\alpha$ and $\delta$ processes have been studied in detail by Floudas et al. ${ }^{15}$ for a SCLCP that presents both smectic and nematic mesophases, using dielectric spectroscopy. They found that increasing temperature strongly shifts the $\alpha$ and $\delta$ processes to higher frequencies. Increasing pressure has similar effects that decreasing temperature, although, in addition, it increases the intensity of the $\alpha$ process due to densification and of the $\delta$ process due to improved order. They concluded that the $\delta$ relaxation was linked to the motion of the mesogenic side chain.

MCLCPs have also received significant attention because they may present thermotropic behavior that lead to a great variety of mesomorphic structures. Their dynamics is more similar to that of semiflexible polymers, showing only local, $\beta$, and glass transition, $\alpha$, relaxations. Nevertheless, there are few systematic studies on the relaxation of this type of polymers, and most of them are focused on polyesters.

Polybibenzoates, $\mathrm{PB}$, with all-methylene spacers represent an ideal model for studying the structure and phase formation of MCLCPs. PBs are formed by biphenyl groups linked by methylene chains; for such all-methylene spacers, the smectic phases formed are quite unstable, and crystallization is fast. The biphenyl group is able to generate smectic mesophases with isotropization temperatures, which generally decrease as the length of the alkylene spacer $(m)$ increases, although a noticeable even-odd effect has been observed. ${ }^{16,17}$ Polybibenzoates with $2 \leq m \leq 10$ form a smectic phase on cooling, followed by a transition into a three-dimensional crystalline phase. However, on heating, only members with $m<7$ exhibit the crystal-smectic transition, while polymers with $m>7$ undergo a monotropic transition directly into the isotropic melt.

Low-ordered smectic mesophases are usually involved in the phase behavior of PBs. Those include SmA or SmC mesophases, where the molecular axes are perpendicular (orthogonal mesophase) or inclined (tilted mesophase), respectively, in relation to the smectic planes.

Frequently, it has been found that the amorphous phase cannot be quenched by supercooling the isotropic melt because the formation of the mesophases is very fast. However, the transformation rate can be controlled by suitable changes in the structure of the flexible spacer. The substitution of the methylene by an ether or the introduction of lateral groups stabilizes the mesophases and decreases the phase transition temperatures. $^{18-20}$

The insertion of side groups and/or ether groups along the spacer helps tuning the stiffness of the polymer chain. The use of diols bearing ether and methyl groups in the synthesis of PBs leads to random copolymer structures that usually favor the stability of the mesophase, the liquid crystallization is slowed down, and sometimes it is possible to quench the isotropic melt to the amorphous state by cooling at not very high rates. ${ }^{21}$

Depending on whether each repetitive unit has one or two methyl lateral substituents, the polymer forms smectic $\mathrm{C}$ or nematic mesophases. Ezquerra et al. have recently studied ${ }^{8}$ one of these copolymers using dielectric relaxation spectroscopy, DR. They found that the copolymer shows an isosbestic frequency, previously described in SCLCPs as they are realigned in electric fields and for long side chain methacrylates during crystallization. ${ }^{22}$ This means that the $\alpha$ process characteristic of the amorphous part is being replaced by a faster $\alpha$ process characteristic of the liquid crystal phase with lower $T_{\mathrm{g}}$.

The SmC mesophase is particularly interesting since, by introducing chiral centers into the structures, it may develop mesophases with ferroelectric properties. ${ }^{23}$ In the case of ferroelectric liquid crystals, Kremer et al. have described a complex dielectric behavior for unaligned and for aligned polymers in terms of the chiral smectic $\mathrm{C}$ phase, namely, Goldstone and soft mode, respectively. ${ }^{24}$

The aim of the present work is to synthesize a new main chain LCP, poly(triethyleneglycol methyl $p, p^{\prime}$-bibenzoate), PTEMeB, where it has been possible to observe, independently, both the amorphous and the liquid crystalline glasses, thus allowing us to analyze the corresponding similarities and/or differences between them. In this polymer, the methyl side groups are in different positions in the spacer than in the polymer studied by Ezquerra et al., ${ }^{8}$ thus modifying the chain flexibility. In the first part of the work, the structural characterization of PTEMeB is done using differential scanning calorimetry (DSC), real-time synchrotron X-ray experiments, and X-ray diffraction in fibers oriented by uniaxial stretching. In the second part, a comprehensive dielectric relaxation study is described, analyzing both the temperature and pressure dependence of the glass transitions of both the isotropic amorphous polymer and the liquid-crystalline mesophase. The kinetics of the phase transition from one state to the other has also been studied.

\section{EXPERIMENTAL SECTION}

2.1. Synthesis. 2.1.1. Materials. Propylene oxide (Fluka) was stirred for $48 \mathrm{~h}$ over $\mathrm{KOH}$ pellets and distilled in a vacuum line prior to being used. Diethylene glycol (Fluka) was purified by distillation at reduced pressure. Dimethyl 1,1'-biphenyl-4,4'dicarboxylate (Aldrich) was purified by recrystallization in chloroform. Titanium(IV) isopropoxide (Aldrich) was used as received.

2.1.2. Techniques. Gas chromatography (GC) analysis was performed by using an Agilent Technologies 6890N Gas Chromatograph equipped with a 5973 quadrupole mass selective detector (Agilent Technologies). A HP-5 ms fusedsilica capillary column $(30 \mathrm{~m} \times 0.25 \mathrm{~mm}$ i.d., $0.25 \mu \mathrm{m}$ film thickness of 5\% phenyl methylpolysiloxane) was employed for diol separation. Helium was the carrier gas, with a gas flow velocity of $0.8 \mathrm{~mL} / \mathrm{min}$. The split ratio was $42: 1$. Injector temperature was $250{ }^{\circ} \mathrm{C}$. The oven temperature program 
consisted of an initial temperature of $130{ }^{\circ} \mathrm{C}$ for $6 \mathrm{~min}$, followed by a temperature gradient of $6{ }^{\circ} \mathrm{C} / \mathrm{min}$ up to $180^{\circ} \mathrm{C}$. The mass spectrometer detector was tuned by maximum sensitive autotune. Solution nuclear magnetic resonance (NMR) spectroscopy measurements were carried out in an Inova-400 spectrometer, using deuterated chloroform as the solvent. The spectra were acquired at room temperature. The intrinsic viscosity was measured in chloroform at $25^{\circ} \mathrm{C}$ with an Ubbelohde viscometer.

2.1.3. Diol Synthesis and Structural Characterization. The diol was synthesized by a protonic acid catalyzed ring-opening reaction of propylene oxide with diethylene glycol. Because propylene oxide is an asymmetrically substituted oxirane, two pathways in the opening of the ring are possible (Figure 1), and
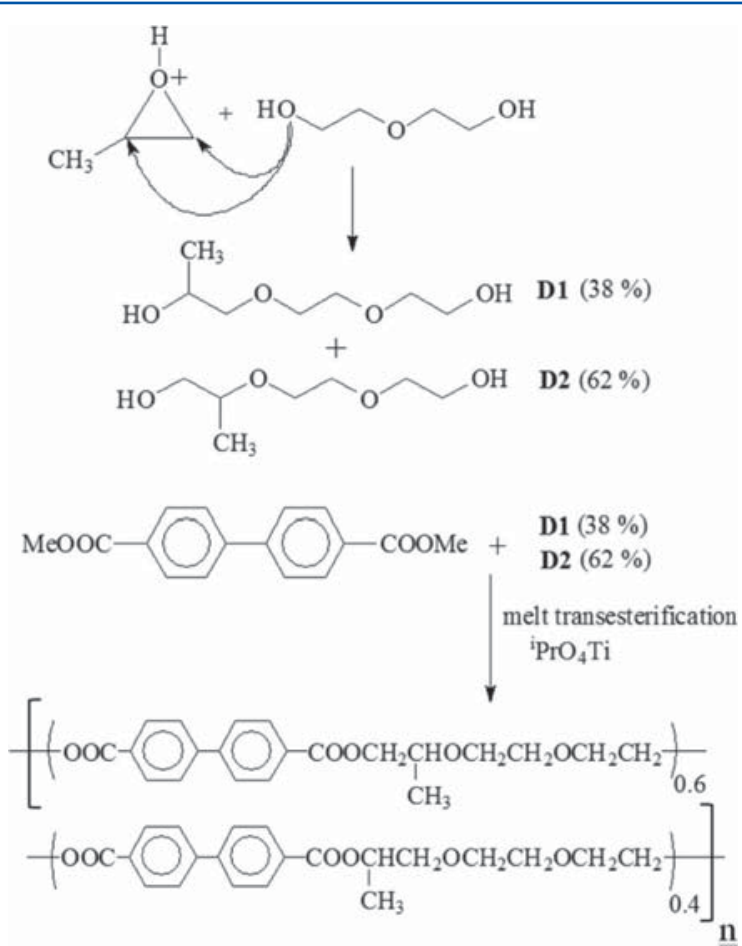

PTEMeB

Figure 1. Synthesis of polybibenzoate PTEMeB.

therefore, a mixture of the isomer ether-diols D1 and D2 can be formed. However, the propylene oxide competes with diethylene glycol in the addition to the protonated oxirane. Thus, the ring-opening with propylene oxide can be reduced by keeping a low instantaneous concentration of oxirane in the reaction mixture. More information about this synthetic route can be found elsewhere. ${ }^{21}$

The experimental procedure was as follows: propylene oxide $(61.6 \mathrm{~g}, 1.06 \mathrm{~mol})$ was added dropwise to a solution of sulfuric acid $(21.0 \mathrm{~g}, 0.21 \mathrm{~mol})$ in diethylene glycol $(430 \mathrm{~g}, 4.05 \mathrm{~mol})$. The reaction was completed after stirring at room temperature under vacuum for $24 \mathrm{~h}$. Subsequently, the mixture was neutralized with sodium carbonate, and the precipitated salt was eliminated by centrifugation. Finally, the unreacted diethylene glycol and oligomers (formed in small quantities) were removed by consecutive distillations under vacuum. The purity and composition of the fractions was checked by gas chromatography (GC). The isomer diols gave separated signals in GC, and the ratio 38:62, with an estimated uncertainty of \pm 2 , was found in the final distilled mixture, in agreement with the composition estimated in the mixture prior to purification.

The identification of the isomers D1 and D2 was based on NMR analysis. The ${ }^{1} \mathrm{H}$ NMR and ${ }^{13} \mathrm{C}$ NMR signals were assigned using COSY, HMQC, and DEPT experiments. Attending to the relative intensity of ${ }^{13} \mathrm{C}$ NMR signals, it was concluded that the proportions of the diols D1 and D2 in the mixture were $\mathrm{D} 1=38 \%$ and $\mathrm{D} 2=62 \%$, in agreement with GC analysis.

${ }^{1} \mathrm{H}$ NMR: $\delta=1.02$ (d, $\left.\mathrm{HOCH}_{2} \mathrm{CH}\left(\mathrm{CH}_{3}\right) \mathrm{O}\right), 1.04$ (d, $\left.\mathrm{HOCH}\left(\mathrm{CH}_{3}\right) \mathrm{CH}_{2} \mathrm{O}\right), 3.18-3.42\left(\mathrm{ABX}, \mathrm{HOCH}\left(\mathrm{CH}_{3}\right) \mathrm{CH}_{2} \mathrm{O}\right)$, 3.44-3.55 (m, $\left.\mathrm{HOCH}_{2} \mathrm{CH}\left(\mathrm{CH}_{3}\right) \mathrm{OCH}_{2}, \mathrm{OCH}_{2} \mathrm{CH}_{2} \mathrm{OH}\right)$, 3.56-3.60 (m, $\left.\mathrm{CH}_{2} \mathrm{OCH}_{2} \mathrm{CH}_{2} \mathrm{OH}, \mathrm{HOCH}\left(\mathrm{CH}_{3}\right) \mathrm{CH}_{2} \mathrm{OCH}_{2}\right)$, 3.60-3.76 (m, OCH $\left.\mathrm{CH}_{2} \mathrm{OH}, \mathrm{HOCH}_{2} \mathrm{CH}\left(\mathrm{CH}_{3}\right) \mathrm{O}\right), 3.89$ (m, $\left.\mathrm{HOCH}\left(\mathrm{CH}_{3}\right) \mathrm{CH}_{2} \mathrm{O}\right)$ ppm.

${ }^{13} \mathrm{C}$ NMR: $\delta=15.8\left(\mathrm{HOCH}_{2} \mathrm{CH}\left(\mathrm{CH}_{3}\right) \mathrm{O}\right), 18.4(\mathrm{HOCH}-$ $\left.\left(\mathrm{CH}_{3}\right) \mathrm{CH}_{2} \mathrm{O}\right), 61.2\left(\mathrm{OCH}_{2} \mathrm{CH}_{2} \mathrm{OH}\right), 65.8\left(\mathrm{HOCH}_{2} \mathrm{CH}-\right.$ $\left.\left(\mathrm{CH}_{3}\right) \mathrm{OCH}_{2}\right), 65.9\left(\mathrm{HOCH}\left(\mathrm{CH}_{3}\right) \mathrm{CH}_{2} \mathrm{O}\right), 67.8\left(\mathrm{HOCH}_{2} \mathrm{CH}-\right.$ $\left.\left(\mathrm{CH}_{3}\right) \mathrm{O}\right), 70.0$ and $70.2\left(\mathrm{HOCH}\left(\mathrm{CH}_{3}\right) \mathrm{CH}_{2} \mathrm{OCH}_{2} \mathrm{CH}_{2}\right), 70.4$ $\left(\mathrm{HOCH}_{2} \mathrm{CH}\left(\mathrm{CH}_{3}\right) \mathrm{OCH}_{2} \mathrm{CH}_{2}\right), 72.5\left(\mathrm{OCH}_{2} \mathrm{CH}_{2} \mathrm{OH}\right), 76.8$ $\left(\mathrm{HOCH}_{2} \mathrm{CH}\left(\mathrm{CH}_{3}\right) \mathrm{O}\right), 76.9\left(\mathrm{HOCH}\left(\mathrm{CH}_{3}\right) \mathrm{CH}_{2} \mathrm{O}\right) \mathrm{ppm}$.

2.1.4. Polymer Synthesis and Characterization. The polymer PTEMeB was synthesized by melt transesterification of dimethyl 1,1'-biphenyl-4,4'-dicarboxylate and the mixture of diols [D1 (38\%) and D2 (62\%)], using a two-stage polycondensation as shown in Figure 1. In the first step, the diester $(33.6 \mathrm{~g}, 0.124 \mathrm{~mol})$ and the mixture of diols $(22.5 \mathrm{~g}$, $0.137 \mathrm{~mol}$ ) were stirred, in the presence of titanium(IV) isopropoxide as a catalyst, at $210{ }^{\circ} \mathrm{C}$ under nitrogen atmosphere for several hours. In a second step, the temperature was then increased up to $250{ }^{\circ} \mathrm{C}$ under reduced pressure $(0.5$ $\mathrm{mmHg}$ ). The polymer was purified by dissolving in hot chloroform and precipitating in excess methanol. Subsequently, it was collected by filtration, washed with methanol, and dried in vacuum.

The chemical structure of the polymer was ascertained by solution ${ }^{13} \mathrm{C}$ and ${ }^{1} \mathrm{H}$ NMR measurements. The signals were assigned using complementary two-dimensional experiments such as COSY and HMQC.

${ }^{1} \mathrm{H}$ NMR: $\delta=1.27$ (d, 3H, Hc), 1.36 (d, 3H, Hc'), 3.603.80 (m, 10H, Hb' $\left.\mathrm{Hd}^{\prime}, \mathrm{Hd}^{\prime}, \mathrm{He}, \mathrm{He}^{\prime}\right), 3.80-3.95$ (m, 5H, Hb, Hf, $\left.\mathrm{Hf}^{\prime}\right), 4.23$ (m, 2H, Ha), 4.47 (m, $\left.4 \mathrm{H}, \mathrm{Hg}, \mathrm{Hg}^{\prime}\right), 5.35$ (m, $\left.1 \mathrm{H}, \mathrm{Ha}^{\prime}\right), 7.50-8.20(\mathrm{~m}, 16 \mathrm{H}$, Harom.) ppm.

${ }^{13} \mathrm{C}$ NMR: $\delta=16.7\left(C c^{\prime}\right), 17.1(C c), 64.2\left(\mathrm{Cg}, \mathrm{Cg}^{\prime}\right), 67.7$ (Ca), $68.8(\mathrm{Cd}), 69.2\left(\mathrm{Cf}, \mathrm{Cf}^{\prime}\right), 70.1\left(\mathrm{Ca}^{\prime}\right), 70.6\left(\mathrm{Ce}^{\prime}\right), 70.8$ $\left(\mathrm{Cd}^{\prime}\right), \quad 70.9$ (Ce), $73.7 \quad\left(\mathrm{Cb}^{\prime}\right), 73.9(\mathrm{Cb}), 127.1$ (CCHCHCCOO), 129.5 (CCHCHCCOO), 130.1 (CCHCHCCOO), 144.2 (CCHCHCCOO), 165.6-166.1 (CCHCHCCOO) ppm.

Figure 2 shows the ${ }^{1} \mathrm{H}$ NMR spectrum of PTEMeB. The polymer composition was determined by comparison of the integration of the signals $c^{\prime}(1.36 \mathrm{ppm})$ and $c(1.27 \mathrm{ppm})$ associated with the lateral methyl group of each structural unit. The proportion found, 40:60, corresponds well, within the experimental error, with the composition of the diol mixture.

A film of the polymer was prepared by compression molding in a Collin Press between hot plates $\left(80^{\circ} \mathrm{C}\right)$ and subsequently cooled to room temperature between water-cooled plates in press. The intrinsic viscosity of PTEMeB was found to be 0.20 $\mathrm{dL} \mathrm{g}^{-1}$, from which $M_{\mathrm{n}}$ a number average molecular weight, was estimated to be 10000 following the same method as that in ref 21 . 


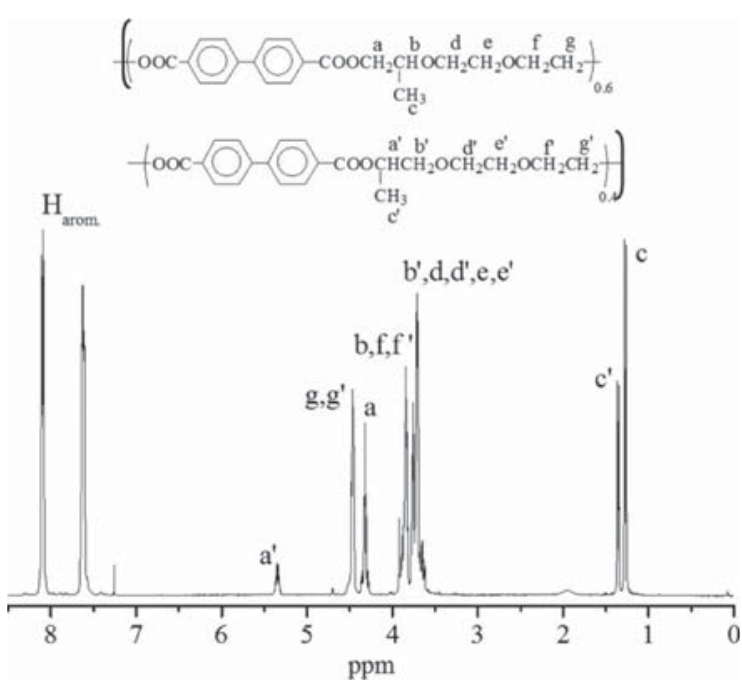

Figure 2. ${ }^{1} \mathrm{H}$ NMR solution spectrum of PTEMeB.

2.2. Techniques. Differential scanning calorimetric (DSC) measurements were carried out under dry nitrogen purge with a TA Instruments Q100 calorimeter connected to a cooling system. The glass transition temperature, $T_{g}$, was considered as the temperature where the increment of the specific heat is onehalf of the total change at the transition. The isotropization

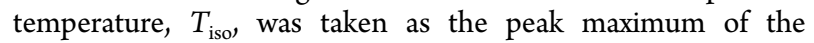
endotherm. About 6-7 mg of sample was encapsulated in an aluminum pan. The following procedure was used: the sample was first melted at $90^{\circ} \mathrm{C}$ for 2 min to erase the thermal history, and then, it was subjected to a cooling/heating cycle at $20^{\circ} \mathrm{C} /$ min. After heating, the sample was cooled down to 30 at 20 ${ }^{\circ} \mathrm{C} / \mathrm{min}$, kept at this temperature for a determined annealing time, and rapidly cooled to $-25^{\circ} \mathrm{C}$, and then, the subsequent heating to $90^{\circ} \mathrm{C}$ at a rate of $20^{\circ} \mathrm{C} / \mathrm{min}$ was registered.

Variable-temperature $\mathrm{X}$-ray diffraction experiments were performed on the Spanish CRG BM16 beamline at ESRF (Grenoble, France). The X-ray beam was monochromatized at the selenium edge $(\lambda=0.098 \mathrm{~nm})$. A MARCCD detector, placed at around $25 \mathrm{~cm}$ from the polymer sample and off beam, was able to cover both the MAXS and WAXS regions (spacings from around 10 to $0.27 \mathrm{~nm}$ ). A Linkam THMS600 stage was used for controlling the temperature. A scanning rate of $8{ }^{\circ} \mathrm{C} /$ min was employed. The scattering patterns were collected in time frames of $15 \mathrm{~s}$, so that we have a temperature resolution of $2{ }^{\circ} \mathrm{C}$ between frames. Crystalline iPP and silver behenate samples were used for the calibration of the detector.

The two-dimensional MAXS/WAXS diffraction patterns were integrated to one-dimensional diffactograms using the FIT2D program of Dr. A. Hammersley of ESRF. The profiles were normalized to the primary beam intensity, and the background from an empty sample was subtracted. The corresponding profiles represent relative intensity units as a function of the reciprocal vector, $s=1 / d$.

Oriented fibers were obtained by uniaxial drawing of dumbbell shaped specimens in a Minimat 2000 dynamometer at $20{ }^{\circ} \mathrm{C}$ and with a crosshead speed of $50 \mathrm{~mm} / \mathrm{min}$, up to a final strain of $300 \%$. The specimens for these experiments were punched out from a film of PTEMeB in the liquid crystalline state. The dimensions of these specimens were $15 \mathrm{~mm}$ long, 3 $\mathrm{mm}$ wide, and $0.7 \mathrm{~mm}$ thick. Actual strain rates were determined from the displacement of parallel ink marks printed $2 \mathrm{~mm}$ apart prior to drawing.

Wide-angle X-ray diffraction photographs of fibers were taken using a flat-plate camera attached to a Phillips $2 \mathrm{~kW}$ tube $\mathrm{X}$-ray generator using nickel-filtered $\mathrm{CuK}_{\alpha}$ radiation. The azimuthal integration of these $\mathrm{X}$-ray photographs was performed in a narrow range of scattering vector around the smectic layer spacing. The origin for the azimuthal angle, $\alpha$, has been taken in the equator (fiber direction in the meridian).

The dielectric experiments were done using an Alpha-N Novocontrol analyzer, operating in the frequency range from $10^{-1}$ to $10^{6} \mathrm{~Hz}$. The samples are parallel-plate capacitors of 30 $\mathrm{mm}$ in diameter (24 $\mathrm{mm}$ for high pressure measurements) and $0.1 \mathrm{~mm}$ thickness maintained by glass fibers acting as spacers. The atmospheric pressure experiments were performed in the isothermal mode using a homemade cell and nitrogen cryostatthermostat that allowed for holding the temperature within $\pm 0.1{ }^{\circ} \mathrm{C}$. The usual warming/cooling rates between two isotherms, with a $10 \mathrm{~min}$ thermostatization period, were approximately $2{ }^{\circ} \mathrm{C} / \mathrm{min}$. For the pressure dependent measurements, up to $300 \mathrm{MPa}$, a Novocontrol High Pressure Test System was used. It consists of a temperature controlled chamber and a hydraulic pressure generation system. The temperature and pressure were controlled within $\pm 0.1{ }^{\circ} \mathrm{C}$ and $\pm 1 \mathrm{MPa}$, respectively. Dried silicon oil (210H Dow Corning) was used as the pressure-transmission medium. The electrodes of the sample capacitor and the electrical connections were sealed with epoxy glue, and the interelectrode material was isolated from the oil by a ring made of natural rubber that transmitted the pressure to the sample (Poisson ratio of natural rubber $\left.^{25}=0.499\right)$. The measurements were made collecting different isobaric dielectric data at a fixed temperature $\left(T_{\mathrm{sp}}\right)$. The experiments were started at the maximum working pressure $\left(p_{\max }\right)$, and the dielectric spectra, $\varepsilon^{*}\left(f ; p_{\mathrm{sp}}, T_{\mathrm{sp}}\right)$, were measured in consecutive steps of decreasing pressure $\left(p_{\mathrm{sp}}\right)$ by opening a venting valve. As the pressure changes are quite fast, they induce temperature changes; thus, an equilibration period of $30 \mathrm{~min}$ was necessary to recover the equilibrium temperature. The thermal history of the sample and the measurements protocols depended on the type of the experiments carried out and will be described in the next section.

\section{RESULTS AND DISCUSSION}

3.1. Calorimetric and X-ray Diffraction Studies. The DSC curves of PTEMeB during a cooling-heating cycle at 20 ${ }^{\circ} \mathrm{C} / \mathrm{min}$ are presented in Figure 3a. They show only the change in the specific heat associated with the glass transition (at around $25^{\circ} \mathrm{C}$ on heating), and no other thermal transitions are observed. Thus, after cooling from the isotropic melt, PTEMeB exhibits an amorphous phase. This has been confirmed by X-ray diffraction experiments. Figure 4a shows that, in the MAXS/ WAXS profile acquired after cooling PTEMeB, only a broad WAXS halo is observed; it is centered at around $0.44 \mathrm{~nm}$ and is associated with the averaged intermolecular distance of polymer chains in an amorphous state. However, when the polymer is annealed at temperatures above the glass transition for a certain time, some ordering is developed in the sample. The diffractogram in Figure $4 \mathrm{~b}$ shows a MAXS diffraction peak at around $2 \mathrm{~nm}$ (and its second order) and an amorphous-like WAXS broad peak. This profile is characteristic of a lowordered smectic mesophase where the sharp MAXS diffraction arises from the smectic layer spacing, and the broad WAXS halo 


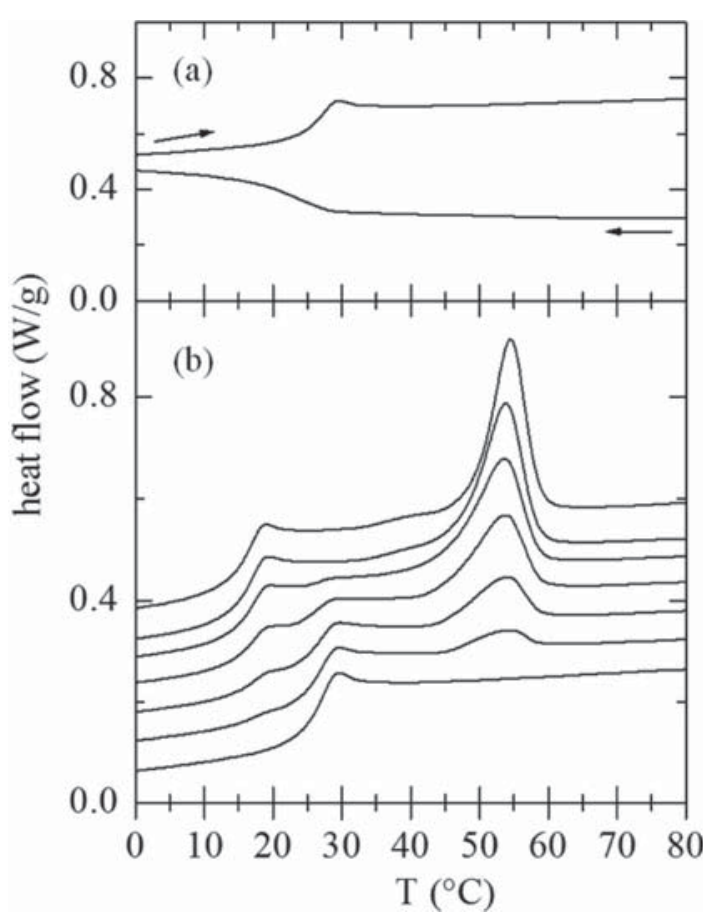

Figure 3. DSC curves corresponding to PTEMeB. (a) Cooling from the isotropic melt (bottom curve) and subsequent heating (top curve); (b) heating curves after annealing the sample at $30{ }^{\circ} \mathrm{C}$ for different times (from bottom to top: $0,60,75,90,105,180$, and $2400 \mathrm{~min}$ ). Scanning rate: $20^{\circ} \mathrm{C} / \mathrm{min}$.

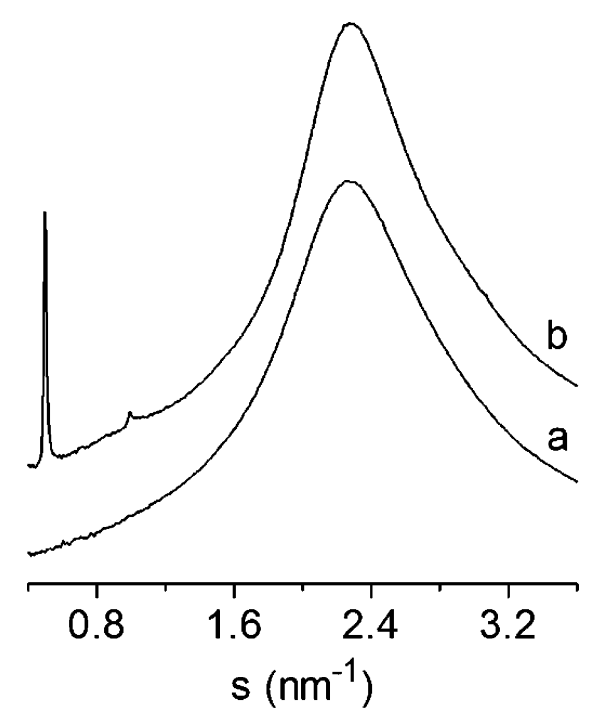

Figure 4. MAXS/WAXS profiles, at $30{ }^{\circ} \mathrm{C}$, corresponding to PTEMeB: (a) sample cooled from the isotropic melt; (b) sample annealed at $30{ }^{\circ} \mathrm{C}$ for $5 \mathrm{~h}$.

indicates the absence of positional order within the smectic layers.

Moreover, an endothermic peak is observed in the DSC heating curve. This fact is shown in Figure $3 b$, where the heating curves after annealing at $30{ }^{\circ} \mathrm{C}$ during different times are displayed (the curves are shifted vertically for clarity). After this annealing, an endotherm is detected near $T_{\text {iso }}=54{ }^{\circ} \mathrm{C}$; its intensity increases with the annealing time, although its position remains almost constant. It is important to notice the presence of a shoulder at around $40{ }^{\circ} \mathrm{C}$ that is more clearly detected in the curves corresponding to the higher annealing times. The origin of this shoulder will be discussed later on. As shown in Figure 5 (lower frame), the isotropization enthalpy,
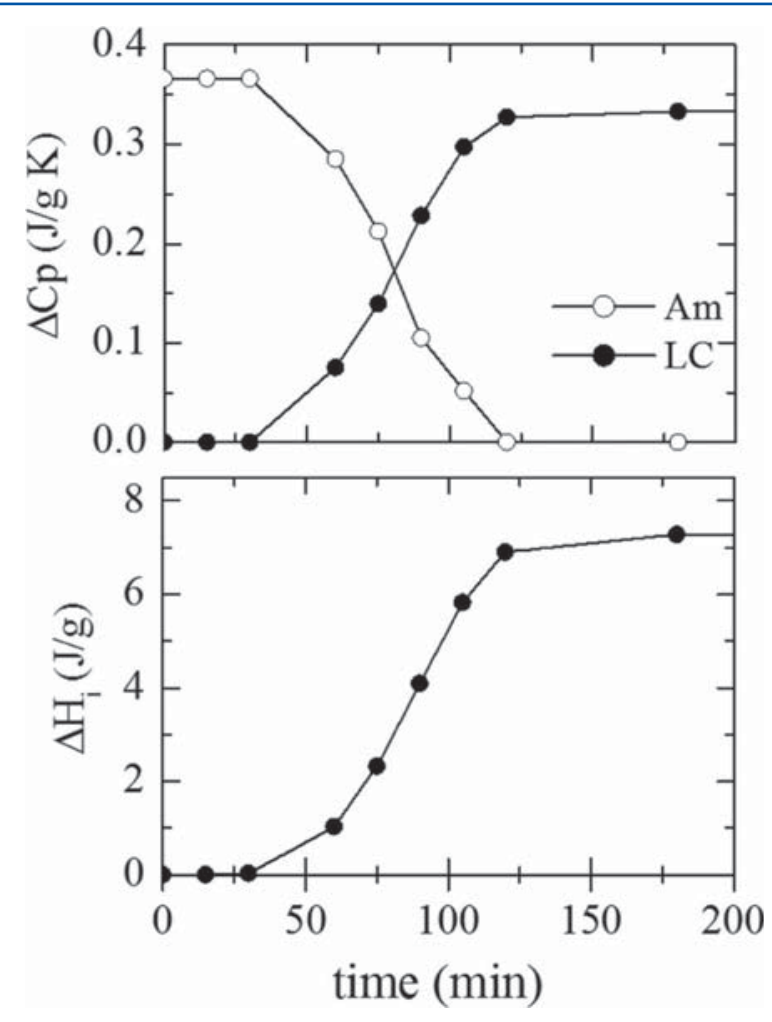

Figure 5. Variation of the increment of specific heat at the two different glass transitions (upper) and variation of the isotropization enthalpy $\Delta H_{\mathrm{i}}$ (lower) as a function of the annealing time for PTEMeB annealed at $30{ }^{\circ} \mathrm{C}$.

$\Delta H_{\mathrm{i}}$, increases up to an asymptotic value close to $7.5 \mathrm{~J} / \mathrm{g}$. This low value of enthalpy change suggests the presence of a lowordered mesophase as deduced from the diffractogram in Figure $4 \mathrm{~b}$.

The extent of the amorphous-liquid crystal transformation achieved during the annealing of PTEMeB is also reflected in the glass transition region. Two separated steps are observed in the DSC curves (Figure 3), centered at 15 and $25{ }^{\circ} \mathrm{C}$, respectively, whose intensities (increment of specific heat) depend on the annealing time. At zero annealing time, only the glass transition at $25^{\circ} \mathrm{C}$, associated with the amorphous phase of PTEMeB, is observed. The intensity of this step decreases with annealing time, in other words, when the content of the mesophase formed increases. At the same time, a new step at 15 ${ }^{\circ} \mathrm{C}$ appears and grows until becoming the only one observed at high annealing times. The variations of the specific heat increments with annealing time at the glass transitions of the two phases, amorphous (am) and liquid crystal (LC), are shown in the upper part of Figure 5. The fact that $\Delta C_{p}$ for the amorphous component goes to zero at high annealing times, when the transformation is complete, suggests that the mesophase formation is a process that reaches almost $100 \%$ of the material volume, in contrast with the low crystallinity attained by regular semicrystalline polymers. 
Variable-temperature X-ray experiments were performed using synchrotron radiation to obtain more information about the thermotropic behavior of PTEMeB. The MAXS/WAXS profiles acquired during the heating of a sample of PTEMeB in the liquid crystalline state are shown in Figure 6. It is observed

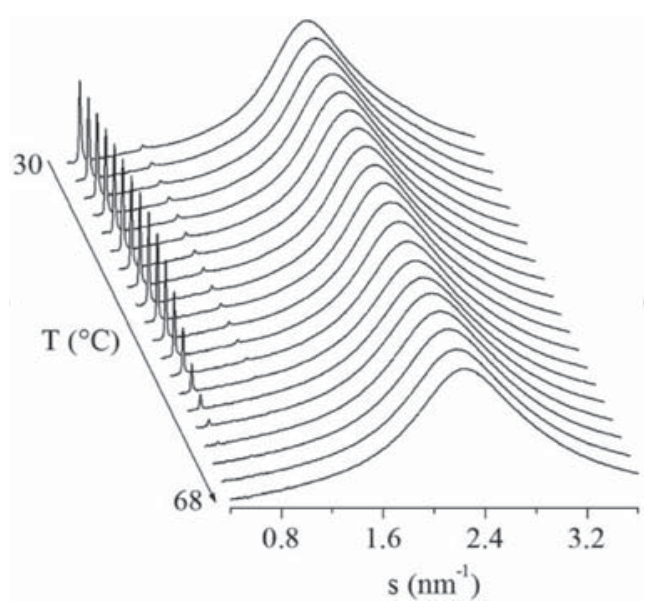

Figure 6. MAXS/WAXS synchrotron profiles during the heating of a sample of PTEMeB annealed at $30^{\circ} \mathrm{C}$. Scanning rate: $8{ }^{\circ} \mathrm{C} / \mathrm{min}$.

that the MAXS peak, attributed to the regular piling of smectic layers, remains approximately constant in position and disappears totally at $65{ }^{\circ} \mathrm{C}$, in agreement with the endotherm observed in the DSC heating curve. However, the isotropization of the mesophase produces a widening and a slight change in position of the WAXS halo, suggesting an increase of the averaged intermolecular distances.

A careful analysis of the MAXS peak has been done in these diffractograms. For clarity, the MAXS profiles corresponding to selected temperatures have been represented in the upper part of Figure 7. It is observed that, at lower temperatures, the peak is noticeably asymmetric, presenting a shoulder at lower spacings (higher $s$ values). On heating the sample up to 45 ${ }^{\circ} \mathrm{C}$, the intensity of the MAXS peak increases and, at the same time, becomes more symmetric. On further heating at temperatures above $48{ }^{\circ} \mathrm{C}$, the peak losses intensity until the isotropization of the sample is attained at $65^{\circ} \mathrm{C}$. The analysis of these profiles has been carried out assuming two overlapped smectic spacings: an intense peak near $0.49 \mathrm{~nm}^{-1}(2.02 \mathrm{~nm}$, peak 1) and a weaker one near $0.51 \mathrm{~nm}^{-1}(1.96 \mathrm{~nm}$, peak 2$)$. As an example, the fitting to two Gaussian functions of the frame acquired at $30{ }^{\circ} \mathrm{C}$ is presented in the lower part of Figure 7. Data derived from this analysis are represented in Figure 8 and compared with the corresponding DSC heating curve. It is observed that the area of peak 2 decreases as $T$ increases, becoming zero at around $48{ }^{\circ} \mathrm{C}$. Simultaneously, the area of peak 1 experiences a parallel increase in such a way that the total area remains constant. This means that the phase associated with peak 2 is transformed into the phase associated with peak 1 . On heating above $48{ }^{\circ} \mathrm{C}$, it is observed that the area of peak 1 decreases, disappearing completely at $65^{\circ} \mathrm{C}$. The derivatives of the area values are also shown in Figure 8. A very good correlation between derivatives and DSC curve is obtained, and two thermal transitions are seen: a weak transition at around $40{ }^{\circ} \mathrm{C}$ and a strong transition centered at $54{ }^{\circ} \mathrm{C}$.

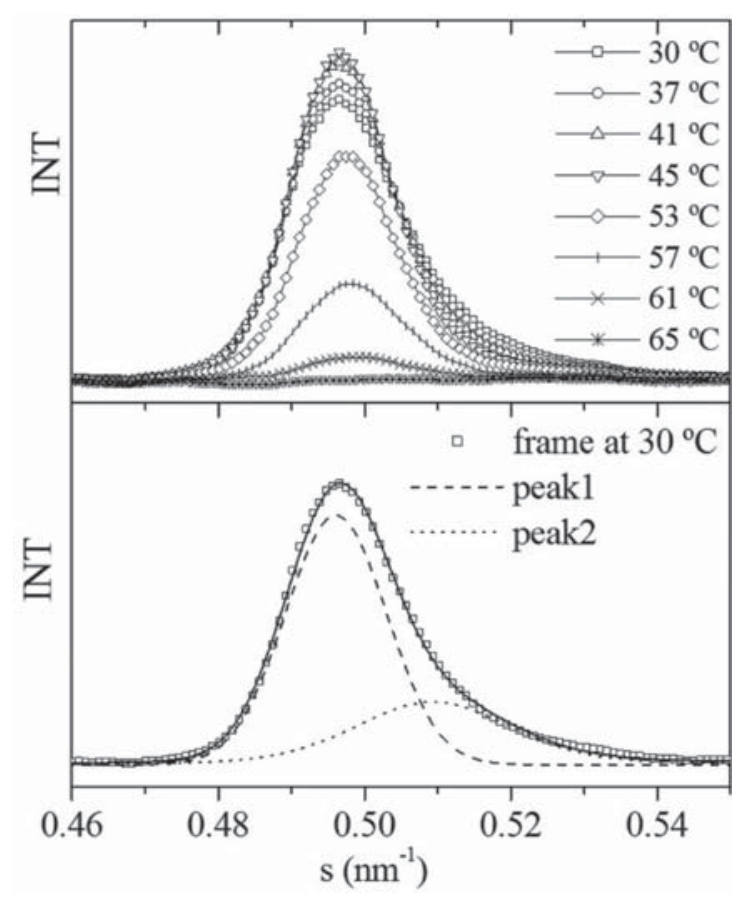

Figure 7. MAXS profiles corresponding to selected temperatures in the heating experiment of Figure 6 (upper part); fitting of the MAXS profile at $30^{\circ} \mathrm{C}$ to two smectic peaks (lower part).

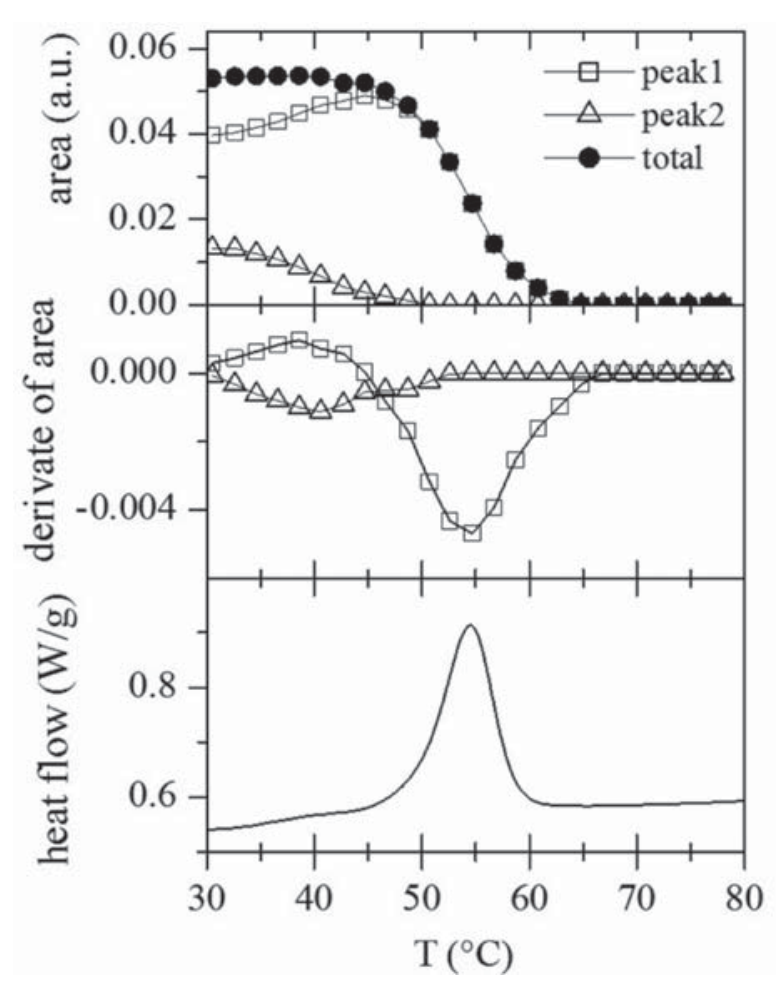

Figure 8. Variation of the area and its derivative of the MAXS peaks as a function of temperature for PTEMeB in the heating experiment of Figure 6. The DSC heating curve of PTEMeB annealed at $30{ }^{\circ} \mathrm{C}$ is presented in the lower part of the figure for comparison.

The phase behavior of poly(triethylene glycol $p, p^{\prime}$ bibenzoate) (PTEB) has been recently studied, ${ }^{26}$ using 
variable-temperature $\mathrm{X}$-ray diffraction, and some interesting features were found. A transition between SmC and SmA mesophases was reported on heating. In addition to the shifting of the smectic peak to higher spacings, also an increase of the height and a narrowing of the peak were found, while the area of the peak remained practically unaltered. Moreover, it was also shown that a subtle transition is detected by DSC measurements. This fact allows one to propose that the liquidcrystalline state of PTEMeB is a combination of two mesophases: an orthogonal SmA mesophase and a tilted $\mathrm{SmC}$ mesophase, the former associated with the peak at higher spacings (peak 1) and the latter with the less intense and broader peak at lower spacings (peak 2). The angle between the molecular axes and the normal to the smectic planes in the $\mathrm{SmC}$ mesophase can be estimated from the reduction of the smectic spacing using the expression $\cos \theta=d_{\theta} / d_{0}$, where $d_{\theta}$ is the smectic spacing of the SmC mesophase and $d_{0}$ is the smectic spacing for a tilt angle equal to zero (SmA mesophase). Considering that $d_{0}$ is $2.02 \mathrm{~nm}$ and that $d_{\theta}$ is $1.96 \mathrm{~nm}$, the angle between the molecular axes is $14^{\circ}$. Assuming that the area of the MAXS peak is proportional to the amount of mesophase, a composition $75 \%$ of $\mathrm{SmA}$ and $25 \%$ of $\mathrm{SmC}$ can be estimated for the PTEMeB sample above analyzed.

Additional information has been obtained from the analysis of a fiber of PTEMeB uniaxially stretched at $20{ }^{\circ} \mathrm{C}$ from the liquid crystalline state. The corresponding $2 \mathrm{D}$ X-ray photograph is shown in the upper part of Figure 9. It can be observed that it is characteristic of a SmC mesophase: the smectic layer reflection is split at both sides of the meridian (fiber direction)
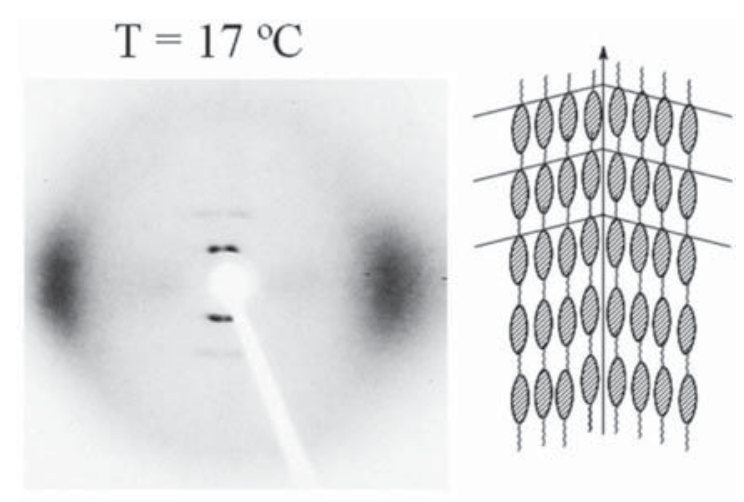

$$
\mathrm{T}=45^{\circ} \mathrm{C}
$$
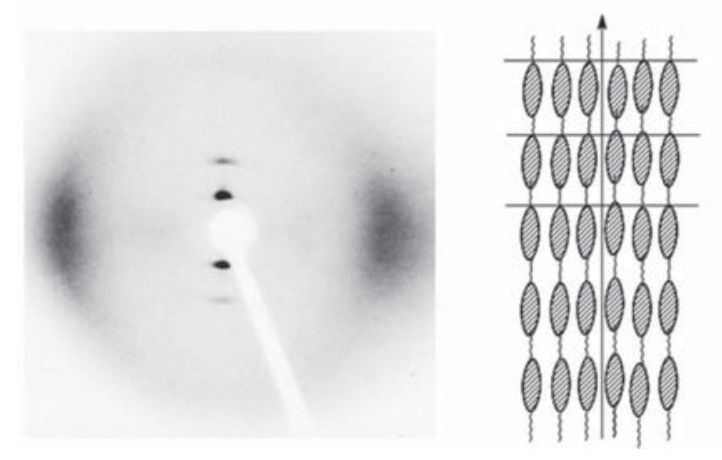

Figure 9. X-ray photographs of PTEMeB at the indicated temperatures after uniaxial stretching of a liquid-crystalline specimen (see Experimental Section). Fiber direction: vertical. The molecular arrangements are also sketched. while the broad wide-angle diffractions are centered on the equator. The azimuthal integration in the region of the smectic spacing is presented in Figure 10. The distribution curve shows

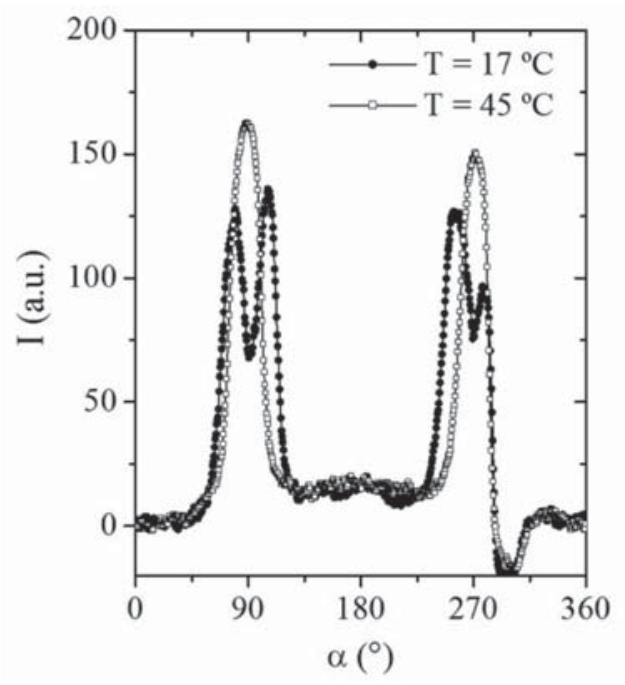

Figure 10. Results for the azimuthal integration in the region of the smectic layer reflection of the X-ray photographs in Figure 9. The origin for the azimuthal angle $\alpha$ has been taken in the equator.

four maxima, the fourth one somewhat covered by the beam stop holder. From the maxima at azimuthal angles of $78^{\circ}$ and $106^{\circ}$ it is deduced that a tilt angle close to $14^{\circ}$ is formed between the normal to the smectic planes and the fiber direction, the same value found for the $\mathrm{SmC}$ phase in the nonoriented sample. However, it seems that, after stretching, the sample becomes $100 \% \mathrm{SmC}$, as deduced from the results of the azimuthal integration: the profile can be fitted just to two peaks centered at angles of $78^{\circ}$ and $106^{\circ}$ with no need for a contribution centered at $90^{\circ}$ ( $\mathrm{SmA}$ mesophase).

When this oriented fiber is heated at $45^{\circ} \mathrm{C}$, slightly above the temperature of the weak transition at $40{ }^{\circ} \mathrm{C}$, the $2 \mathrm{D} \mathrm{X}$-ray photograph (lower picture in Figure 9) is rather different. It corresponds to the profile of $100 \%$ orthogonal SmA mesophase: the smectic layer reflections are on the meridian, showing two maxima at azimuthal angles of $90^{\circ}$ and $270^{\circ}$ (see Figure 10), whereas the broad wide-angle diffractions are centered on the equator. Then, these results confirm the assignment of the weak endotherm close to $T_{\mathrm{SmC}-\mathrm{A}}=40{ }^{\circ} \mathrm{C}$ to a SmC-SmA transition (sketches of the possible molecular arrangements corresponding to the two kinds of mesophases are shown in Figure 9). Interestingly, this transformation in the fiber is completely reversible: after heating at $45^{\circ} \mathrm{C}$, the fiber was cooled down to room temperature, and the corresponding 2D X-ray photograph was identical to that observed for the original fiber, i.e., $100 \% \mathrm{SmC}$ mesophase.

As described above, PTEMeB is a liquid crystalline polymer with a rather slow rate of mesophase formation, thus this polymer can be easily quenched into the glassy amorphous state. Therefore, both the pure amorphous phase and the smectic mesophase of PTEMeB can be analyzed independently in samples with the appropriate thermal history: the amorphous phase was obtained by quenching from the isotropic melt, whereas the mesophase was obtained by annealing at temperature above the amorphous glass transition (and below the isotropization temperature). These two phases show 
different calorimetric glass transitions temperatures: $T_{\mathrm{gAm}}=25$ ${ }^{\circ} \mathrm{C}$ for the amorphous one, and $T_{\mathrm{gLC}}=15{ }^{\circ} \mathrm{C}$ for the mesophase. The existence of two glass transitions for the liquid crystalline and amorphous states in semiflexible polybibenzoates, the former laying at lower temperatures than the later, has been already reported in previous studies based on DSC, dielectric spectroscopy, and/or dynamic-mechanic measurements, as shown in the Introduction.

3.2. Dielectric Relaxation Study. We have studied the temperature and pressure dependence of the $\alpha$ process of PTEMeB in the isotropic liquid as well as in the smectic phases. We also have analyzed the kinetics of the phase transition from one state to another.

3.2.1. Dynamic Glass Transition of the Smectic and Isotropic Phases. The first of the samples studied was denoted as PTEMeB-LC. Starting from the molten state $\left(T>T_{\text {iso }}\right)$, it was suddenly cooled down to $30{ }^{\circ} \mathrm{C}\left(T>T_{\text {gAm }}\right)$ by immersion in liquid nitrogen, and afterward, it was annealed at this temperature $\left(30^{\circ} \mathrm{C}\right)$ for two days. This thermal pretreatment ensured that the material reached the liquid crystalline state (see discussion of Figure 3 in section 3.1). Subsequently, it was cooled down to $19^{\circ} \mathrm{C}$ (at $\left.-2{ }^{\circ} \mathrm{C} / \mathrm{min}\right)$, then, starting from that temperature, a set of dielectric permittivity isotherms were measured while increasing the temperature in steps of $3{ }^{\circ} \mathrm{C}$ up to $76{ }^{\circ} \mathrm{C}\left(T>T_{\text {iso }}\right)$. A few selected experimental curves are shown in Figure 11a, and it is observed that they exhibit a main peak related to the $\alpha$ relaxation, which shifts monotonically to higher frequencies as the temperature is increased up to $46^{\circ} \mathrm{C}$; then, it keeps almost constant up to $52{ }^{\circ} \mathrm{C}$, and later on, it shifts to higher frequencies again. This anomalous behavior is related to the different phase transitions (see section 3.1). Therefore, the $\alpha$ relaxation for temperatures above the isotropization is related to the isotropic fluid (denoted as $\alpha_{\text {iso }}$ ), and below $T_{\text {iso, }}$ it is assigned to the liquid crystalline phase (namely, $\alpha_{\mathrm{LC}}$ ). This one can be composed of SmA and SmC mesophases, and the possible influence of the SmC-SmA transition on the dynamics will be discussed later.

The second sample, named PTEMeB-Iso, was directly measured on cooling from the isotropic liquid phase at 78 ${ }^{\circ} \mathrm{C}$, down to $32{ }^{\circ} \mathrm{C}$ in steps of $1{ }^{\circ} \mathrm{C}$. The main peak of the curves shown in Figure $11 \mathrm{~b}$ at temperatures above $T_{\text {iso }}$ represents the $\alpha_{\text {iso }}$ relaxation. Moreover, the isotherms around and below $T_{\text {iso }}$ do not undergo any shifting and/or broadening of the relaxation peaks as in the case of PTEMeB-LC. It seems that, at the applied cooling conditions and even considering the thermostatic stages, the isotropic liquid phase has been supercooled. Therefore, the peaks of Figure $11 \mathrm{~b}$ correspond to the $\alpha_{\text {iso }}$ relaxation for the whole temperature range. The results in Figures 3 and 5 indicate that crystallization times higher than $30 \mathrm{~min}$ are needed to obtain the mesophases.

The analysis of the dielectric loss curves has been made by fitting the experimental results to the general equation

$$
\begin{aligned}
\varepsilon^{\prime \prime}(\omega)= & \frac{\sigma^{s}}{\left(\varepsilon_{0} \omega\right)^{s}}+\operatorname{Im}\left\{\sum_{k} \frac{\Delta \varepsilon_{k}}{\left[1+\left(\mathrm{i} \omega \tau_{\mathrm{HN}, k}\right)^{a_{k}}\right]^{b_{k}}}\right. \\
& \left.+\frac{\Delta \varepsilon_{\beta}}{1+\left(\mathrm{i} \omega \tau_{\mathrm{CC}, \beta}\right)^{a_{\beta}}}\right\}
\end{aligned}
$$

where $\omega=2 \pi f$ is the angular frequency, $f$ is the frequency, and $\varepsilon_{0}$ is the dielectric permittivity of the vacuum. The first term of the right-hand side accounts for the ionic conductivity $(\sigma)$, the
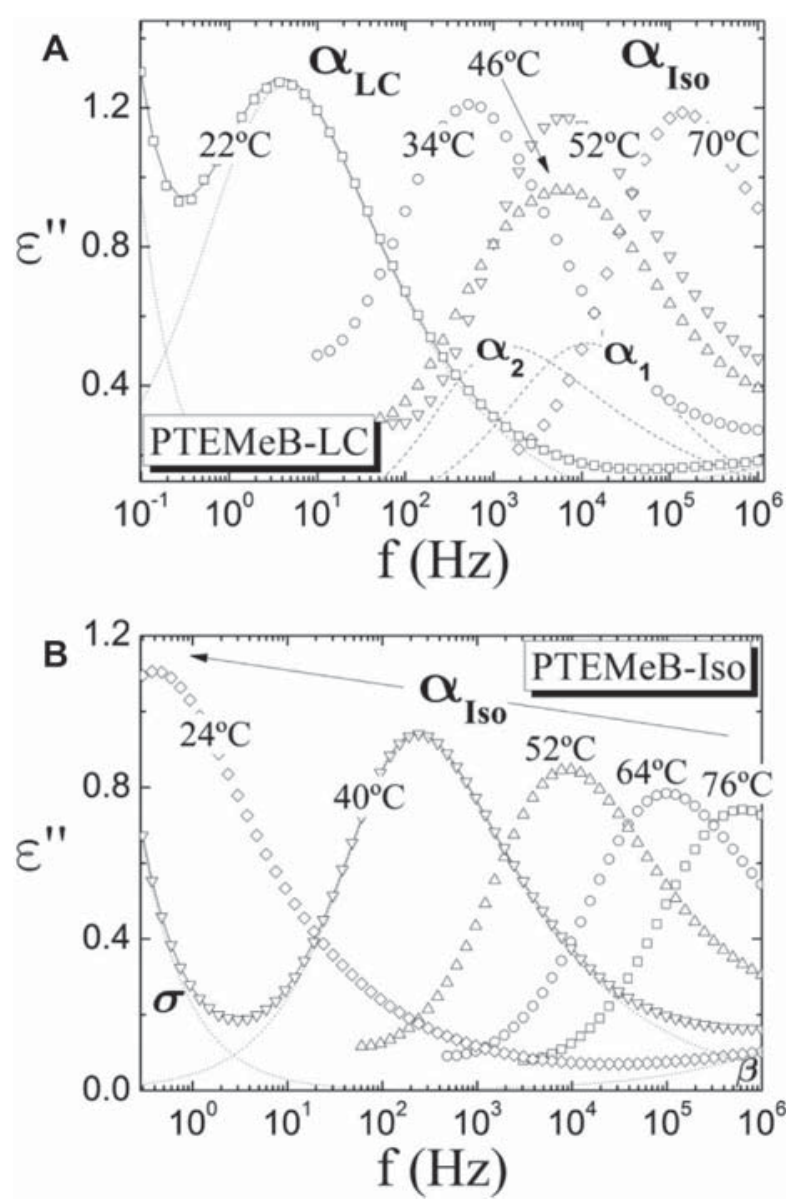

Figure 11. Selected dielectric loss curves (symbols) for PTEMeB as a function of frequency for different temperatures. (a) Related to sample PTEMeB-LC, remarks the $\alpha_{\mathrm{LC}}$ relaxation peak for the 22 and $34{ }^{\circ} \mathrm{C}$

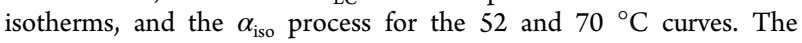
continuous line is the fitting of the $22{ }^{\circ} \mathrm{C}$ curve to eq 1 , using a $\mathrm{HN}$ peak, the conductivity term, and a CC wing (dotted lines). The dashed peaks are related to the intermediate isotherm $\left(46^{\circ} \mathrm{C}\right)$ and represent the contribution of two $\alpha_{k}$ processes $(k=1,2)$, according to the $\mathrm{HN}$ functions extracted from the fitting to eq 1. (b) Related to sample PTEMeB-Iso, measured on cooling, shows the $\alpha_{\text {iso }}$ process for all the temperatures. The line is the fitting of the $40{ }^{\circ} \mathrm{C}$ isotherm to eq 1 with the $\sigma, \alpha_{\text {iso, }}$ and $\beta$ contributions (dashed lines).

strong growth of the imaginary part of the permittivity at low frequencies (see Figure 11). The deviation of the exponent $0 \leq$ $s \leq 1$ from the unity (d.c. conductivity) represents the effect of blocking phenomena in the migration of charge carriers. The terms inside the imaginary part function are used to describe, in the most general case, all the relaxations involved. The Havriliak-Negami models (HN) describe the $\alpha$ peaks ( $k$ index), with $\Delta \varepsilon$ being the dielectric strength (i.e., the difference of the real part of the dielectric permittivity between the relaxed and unrelaxed state); $\tau_{\mathrm{HN}}$ is the parametric relaxation time, and $a$ and $b(0 \leq a, a b \leq 1)$ are the shape exponents related to the width and asymmetry of the relaxation. ${ }^{27}$ At temperatures below $40{ }^{\circ} \mathrm{C}$ and above or equal to $49^{\circ} \mathrm{C}$, a single $\mathrm{HN}$ term has been used to fit the main peak, related to the $\alpha_{\mathrm{LC}}$ process ( $k=$ LC in the eq 1) and to the $\alpha_{\text {iso }}$ mode ( $k=$ iso in the eq 1$)$. For the temperatures $40 \leq T /{ }^{\circ} \mathrm{C}<49$, it was necessary to use two HN functions (namely, $k=1,2$ in eq 1). To illustrate the 
different scenarios, see the fitting examples of the Figure 11. Finally, the last term of eq 1 is a Cole-Cole function with $\Delta \varepsilon$ being the dielectric strength, $\tau_{\mathrm{CC}}$ the relaxation time, and $a(0 \leq$ $a \leq 1)$ the broadening exponent. ${ }^{28}$ This function represents the influence of the left flank of the $\beta$ relaxation on the high frequency part of the permittivity (see Figure 11). We have fixed the values of $\tau_{\mathrm{CC}}$ and $a$ because the peak of this process is outside the frequency window. These parameters were extrapolated from the tendency of low temperatures obtained in exploratory experiments of secondary processes (results not shown).

Following the procedure detailed above, it was possible to fit all the dielectric loss curves for both experiments (PTEMeB-LC and PTEMeB-Iso) and to build the relaxation map for the $\alpha$ relaxation modes (Iso, LC, 1, and 2). The relaxation time can be defined from the maximum of the peaks and calculated from the parametric time and the shape exponents, as in a previous work. $^{29}$

Figure 12 shows the Arrhenius plot for both modes and samples. For PTEMeB-LC, the $\alpha_{\mathrm{LC}}$ is faster than the $\alpha_{\text {iso }}$

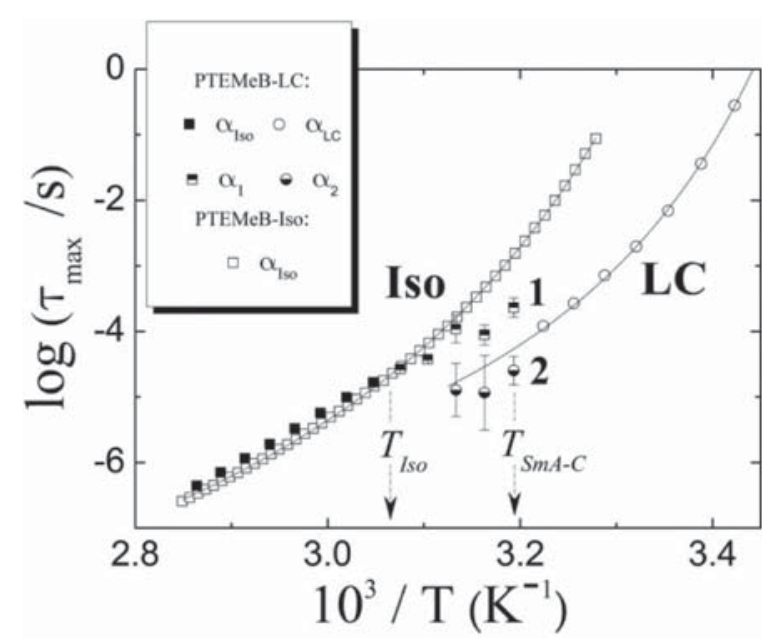

Figure 12. Arrhenius plot of the relaxation times of the $\alpha$ processes (LC, Iso, 1, and 2) for both samples (PTEMeB-LC and PTEMeB-Iso). The lines represent the VFT fits, i.e., eq 2; in the case of $\alpha_{\mathrm{LC}}$, only the points at $T<T_{\mathrm{SmA}-\mathrm{C}}$ have been used in the fitting procedure.

relaxation, and there is a smooth transition between both behaviors of the apparent relaxation time that corresponds to the transition region. The fact that the relaxation times are smaller in the mesophase than in the isotropic liquid is consistent with fact that $T_{\mathrm{gLC}}<T_{\mathrm{gAm}}$. From the calorimetric experiments, it can be considered that the transition region begins at the transition temperature between smectic phases (SmC-A) and ends at the isotropization one, i.e., from 40 to $54{ }^{\circ} \mathrm{C}$ (see section 3.1). The initial part of this interval corresponds to the transition from $\mathrm{SmC}$ to $\mathrm{SmA}$, while at temperatures higher than around $45{ }^{\circ} \mathrm{C}$, the isotropization endotherm begins. However, the transition region of the dielectric results can be defined considering the following behavior: first, the range where the dielectric loss curves have been fitted with two contributions $\left(40-49{ }^{\circ} \mathrm{C}\right.$ ) and, second, the isotherms in $49-55^{\circ} \mathrm{C}$ where the relaxation time of the iso mode of the PTEMeB-LC sample tends to converge to the same as that of the PTEMeB-Iso sample (see Figure 12).
However, the calorimetric experiments were performed by heating at $10{ }^{\circ} \mathrm{C} / \mathrm{min}$, while in the dielectric ones, the overall heating rate is much smaller, so that all the transition regions will be shifted to slightly lower temperatures.

From all the above, it can be stated that both ranges (DSC, $40-54{ }^{\circ} \mathrm{C}$; dielectric, $40-55{ }^{\circ} \mathrm{C}$ ) are similar. For PTEMeB-Iso, the $\alpha_{\text {iso }}$ relaxation time increases smoothly on cooling to the low temperature region, thus confirming (within the experimental uncertainties) the above-mentioned supercooling of the liquid phase. This conclusion is supported by the equal times for both samples in the high temperature region.

The $T$-dependence of the $\alpha$ relaxation times tendencies can be described by a VFT equation:

$$
\tau_{\max }=\tau_{0, \mathrm{VFT}} \exp \left(\frac{D T_{\mathrm{V}}}{T-T_{\mathrm{V}}}\right)
$$

where $\tau_{0, \mathrm{VFT}}$ is a pre-exponential time, $D$ is the strength parameter that measures the deviation from the Arrhenius behavior, and $T_{\mathrm{V}}$ is the Vogel temperature or ideal glass transition. The lines in Figure 12 are the fittings to eq 2. The fitting parameters take the following values: $\log \tau_{0, \mathrm{VFT} \text {,iso }}=$ $-11.0 \pm 0.5$ and $D_{\text {iso }}=3.2 \pm 0.4$ for the iso mode; and log $\tau_{0, \mathrm{VFT}, \mathrm{LC}}=-9.0 \pm 0.5$ and $D_{\mathrm{LC}}=1.8 \pm 0.4$ for the LC process, which reveals a fragile character of the amorphous and liquid crystalline glass formers ${ }^{30}(D<10)$. However, the same value for the Vogel temperature $T_{\mathrm{V}, k}=268 \pm 1 \mathrm{~K}$ describes correctly the trend for both modes $(k=$ iso, LC). The dynamic glass transition temperatures, defined as $T_{\mathrm{g}}^{\mathrm{D}}=T(\tau=100 \mathrm{~s})$, are the following: $T_{\mathrm{g}}^{\mathrm{D}}$ (iso) $=24{ }^{\circ} \mathrm{C}$ and $T_{\mathrm{g}}^{\mathrm{D}}(\mathrm{LC})=14{ }^{\circ} \mathrm{C}$ that agree with the DSC values (see section 3.1). As it can be seen in Figure 12, mode 2 is slightly faster than the LC process that appears at lower temperatures (note that the extrapolated VFT curve of $\tau_{\mathrm{LC}}$ vs $T$ overestimates the last three points); in contrast, mode 1 is slower than LC. Following the discussion of the temperature dependent diffractograms of Figure 7 (see section 3.1), the transient mode 2 can be assigned to the dynamics of the $\alpha$ relaxation of the SmC phase as it transforms into the SmA phase, whereas mode 1 is related to the SmA phase and its gradual isotropization. In other words, the $\alpha$ relaxation of the LC phase, coexistence of smectic A and C phases, is defined at $T<T_{\mathrm{SmC}-\mathrm{A}}$ as $\alpha_{\mathrm{LC}}$ and described with a single $\mathrm{HN}$ function. On the contrary, when $T>T_{\text {SmC-A }}$, the transition between mesophases begins, and also the isotropization and the $\alpha$ relaxation are defined as a superposition of two $\mathrm{HN}$ processes ( 1 and 2 ). In the temperature range $45^{\circ} \mathrm{C}<T<$ $T_{\text {iso }}$, the pure SmA mesophase is melting into the isotropic liquid, as indicated by the slowdown of the relaxation rate. In this situation, the $\alpha$ peak is not wide enough to be described as a sum of two processes. Curiously, the rest of the parameters (the total dielectric strength and the shape exponents) do not vary significantly in the temperature range studied. Particularly, the approximation $\Delta \varepsilon_{\text {iso }} \approx \Delta \varepsilon_{\mathrm{LC}} \approx \Delta \varepsilon_{1}+\Delta \varepsilon_{2} \approx 5$ is always fulfilled. Finally, the exponents have the values $a_{\text {iso }} \approx 0.8, b_{\text {iso }} \approx$ $0.4, a_{\mathrm{LC}} \approx a_{1} \approx 0.6, b_{\mathrm{LC}} \approx b_{1} \approx 0.7, a_{2} \approx 0.7$, and $b_{2} \approx 0.3$.

In summary, two $\alpha$ relaxations have been detected that correspond to the dynamic glass transition of the amorphous phase (isotropic liquid) and to the one of the liquid crystal phase (smectic), respectively. The first, denoted as $\alpha_{\text {iso }}$ is observed at high temperatures after heating the liquid crystal above the isotropization temperature. It can be studied at low temperatures in the supercooled state after cooling the sample at normal cooling rates. One might naively believe that the 
trapped flexible chains between smectic planes have reduced mobility compared to a totally amorphous sample. Indeed, this has been found to be the case for SCLCPs where the $\alpha_{\mathrm{LC}}$ process is associated to the dynamics of the mesogenic side chain. ${ }^{15}$ However, in the case of PTEMeB, the second transition, $\alpha_{\mathrm{LC}}$, was found to be faster. Ahumada et al. proposed an explanation to this apparent contradiction based on the relaxation mechanism of both phases. ${ }^{31}$ In the isotropic case, the $\alpha_{\text {iso }}$ relaxation is attributed to conformational changes derived from segmental motions of the chains, whereas in the smectic phase, the $\alpha_{\mathrm{LC}}$ mode can be related to rotational and translational motions of elongated chains that require smaller free volume, thus leading to lower glass transition temperatures.

3.2.2. Isothermal Formation of the Liquid Crystal Phase. In order to study the transformation from the amorphous phase (supercooled liquid) to the liquid crystal phase, the following experiment was done in parallel to the DSC experiment of Figure $3 \mathrm{~b}$. First, the sample capacitor is rapidly cooled from the molten state to the amorphous phase by immersion in liquid nitrogen. Immediately afterward, the sample was introduced in the atmospheric pressure cell previously stabilized at $30{ }^{\circ} \mathrm{C}$. Finally, the frequency dependent dielectric loss was measured at this temperature and different annealing times, which allowed us to analyze the kinetics of the phase transformation following the $\alpha$ relaxation change. The results are summarized in Figure 13. Initially, only one peak is present: the $\alpha_{\text {iso }}$ process.

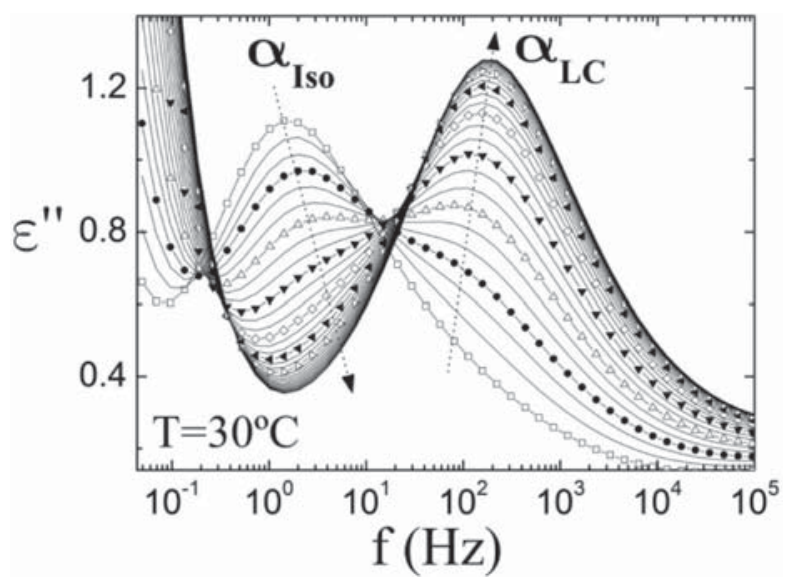

Figure 13. Real time dielectric loss during the isothermal transformation: Isotropic liquid to liquid crystal ( $\mathrm{SmA}$ and $\mathrm{C}$ ). The temperature is $30^{\circ} \mathrm{C}$ and the curves are $20 \mathrm{~min}$ apart. The arrows indicate the time evolution of the $\alpha_{\text {iso }}$ and $\alpha_{\mathrm{LC}}$ peaks. The symbols highlight the first six hours of curves.

At higher times, this mode decreases, and a new peak appears at higher frequencies, which is related to the $\alpha_{\mathrm{LC}}$ relaxation. From the qualitative behavior of the loss curves of Figure 13, we can infer two stages in the kinetics. The first six hours (fast stage) represent a relatively fast decay of the $\alpha_{\text {iso }}$ mode and the corresponding increase of the $\alpha_{\mathrm{LC}}$ mode. Given the overlapping of the curves at long times, the second stage consists of a slow intensity exchange between the two $\alpha$ peaks. Simultaneous dielectric and X-ray diffraction experiments of a similar system have shown that the first stage (fast) is related to the smectic layer formation and the second one to the increase of order within smectic domains. ${ }^{8,32}$

Equation 1 can be used to fit all the isochrones considering the following constraints. For the first curve, only one HN was used to describe the $\alpha_{\text {iso }}$ mode, whereas for longer times, two $\mathrm{HN}$ functions describe the competitive $\alpha_{\text {iso }}$ and $\alpha_{\mathrm{LC}}$ processes; the peaks of the final isochrones can be also described with only one broad HN function. To take into account the left wing of the secondary relaxations, we have fixed the CC parametric time and the shape exponent assuming that the $\beta$ processes does not change during the phase transformation. This last assumption is not generally true; indeed the secondary processes are good probes to follow the relationship between structural changes and the dynamics of crystalline polymers. ${ }^{33}$ In the current case, the monitorization of the secondary peaks in the phase transformation region is not possible because these relaxations are outside the experimental window. However, because of the large separation between $\alpha$ and $\beta$ processes (at least five decades), an eventual change in the characteristic time of $\beta$ would not affect significantly the $\alpha$ relaxations. Therefore, the assumptions made fixing the mentioned parameters of the secondary relaxations can be considered acceptable in our case. Finally, it has to be mentioned that the conductivity is higher in the mesophase than in the isotropic liquid (see the increase of the permittivity at low frequencies with time in Figure 13). This fact can be understood considering that the ion hopping between ordered smectic layers is easier compared to a random motion in a disordered phase.

Figure 14 shows the time evolution of the dielectric strength of the $\alpha$ relaxations. The experimental procedure did not allow

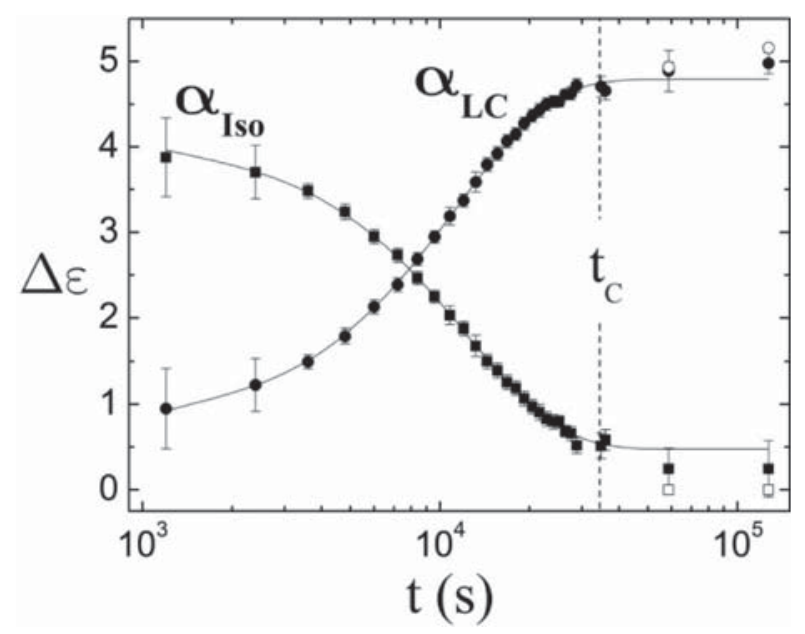

Figure 14. Temporal evolution of the $\alpha_{\text {iso }}$ and $\alpha_{\mathrm{LC}}$ mode dielectric strengths during the liquid crystallization process at $30{ }^{\circ} \mathrm{C}$. The lines are fittings to eqs $3 \mathrm{a}$ and $3 \mathrm{~b}$ for $t<t_{\mathrm{C}}$, where the latter is the estimated time of the smectic layer formation. The open symbols represent equivalent fittings when fixing $\Delta \varepsilon_{\text {iso }}=0$.

us to accurately determine the starting point defined as the elapsed time between the moment immediately after the sample is quenched $(t=0)$ and the time when the first loss curve is measured. An estimation of this time could shift the kinetic curves; thus, only the loss curves for times equal or higher than $20 \mathrm{~min}$, far enough from the starting point, will be discussed. The Kirkwood-Fröhlich theory states that the proportionality constant between the dielectric strength, $\Delta \varepsilon$, and the dipole density is the $g$ correlation factor, ${ }^{34}$ which is related to the correlation between interacting dipole moment vectors, i.e., $\left\langle\sum \boldsymbol{\mu}_{i} \boldsymbol{\mu}_{j}\right\rangle$. The fact that, for the present system, $\Delta \varepsilon_{\text {iso }}(t \rightarrow 0)<\Delta \varepsilon_{\mathrm{LC}}(t \rightarrow \infty)$ can be due to differences in the 
number of dipoles or alternatively on the values of the $g$ factor of the different phases. The first reason is unlikely since the mass of the sample is constant, whereas the latter seems more plausible because the parallel arrangement of the smectic order could favor the correlation between dipole vectors, and therefore a higher $g$ value in the mesophase.

As above-mentioned, two $\alpha$ peaks have been used to describe the loss curves in the entire time span. Nevertheless, fittings to either one or two $\mathrm{HN}$ functions for the longest time peak give almost indistinguishable results (see the points with $\Delta \varepsilon_{\text {iso }}=0$ in Figure 14). This fact was used to estimate the time $t_{\mathrm{C}} \approx 2 \times 10^{4}$ $s$ that separates the two stages of the kinetic phenomenon. The dielectric strength trends for both modes in the fast stage (smectic phase formation, $t<t_{\mathrm{C}}$ ) can be fitted to Avrami models. ${ }^{35,36}$ That is, for the $\alpha_{\text {iso }}$ mode,

$$
\Delta \varepsilon_{\text {iso }}=A_{\text {iso }} \exp \left[-\left(\frac{t}{\tau_{\mathrm{C}, \text { iso }}}\right)^{n_{\text {iso }}}\right]+A_{\text {iso }, 0}
$$

and for the $\alpha_{L C}$ mode,

$$
\Delta \varepsilon_{\mathrm{LC}}=A_{\mathrm{LC}}\left\{1-\exp \left[-\left(\frac{t}{\tau_{\mathrm{C}, \mathrm{LC}}}\right)^{n_{\mathrm{LC}}}\right]\right\}+A_{\mathrm{LC}, 0}
$$

where, $A_{k}$ and $A_{k, 0}$ describe the asymptotic values of the dielectric strengths, $\tau_{\mathrm{C}, k}$ are the time scale of the processes accounting for both the nucleation kinetics and growth rates, and the dimensionless power $n_{k}$ depends on crystal morphology and crystallization mechanism ( $k=$ iso, LC). The intensity parameters have different values (uncertainty $\approx \pm 0.5$ ): $A_{\text {iso }}=$ 3.60, $A_{\mathrm{iso}, 0}=0.47$ and $A_{\mathrm{LC}}=4.10, A_{\mathrm{LC}, 0}=0.75$; while the characteristic time $\tau_{\mathrm{C}, k}=(1.2 \pm 0.3) \cdot 10^{4} \mathrm{~s}$ and the exponent $n_{k}$ $=1.35 \pm 0.05$ are the same for both modes. This confirms that there is only one kinetic phenomenon responsible for the exchange between modes. The time $\tau_{\mathrm{C}}$ has almost the same value as the estimated total time, $t_{\mathrm{C}}\left(10^{4} \mathrm{~s}\right)$ (see above). The value of the exponent $\sim 1$ suggests an athermal nucleation scheme, i.e., all the nucleation sites appear simultaneously and are randomly distributed within the melt; followed by the unidimensional growing (needle-like crystals) of the active nuclei. $^{37}$

The isosbestic points are defined as the specific frequencies where the permittivity is constant during the time evolution (i.e., the species have the same permittivity during the transformation). Inspecting the row data of Figure 13, two crossing points of the curves exist, one at low frequencies (around $0.2 \mathrm{~Hz}$ ) and another at higher frequencies $(20 \mathrm{~Hz}$ ). The high-frequency point is related to the dielectric strength exchange during the phase transformation, and the lowfrequency one gives information about the conductive exchange. However, these two points are not really constant but slightly shift to higher frequencies as time increases. This conclusion is important because there is some controversy in the literature on whether the first order transitions are isochronal or not (similar behavior is found in another small liquid crystals $\left.{ }^{38,39}\right)$. This effect is also detected in the time evolution of the maxima of the $\alpha$ peaks. The plots of $\tau_{\max , k}$ vs time show decreasing trends (see Figure 15). Therefore, it can be concluded that both modes, $\alpha_{\text {iso }}$ and $\alpha_{\mathrm{LC}}$, become faster (shifting almost a decade to higher frequencies) as the phase transition progresses. When plotting the inverse of the isosbestic frequency versus time (e.g., of the high frequency
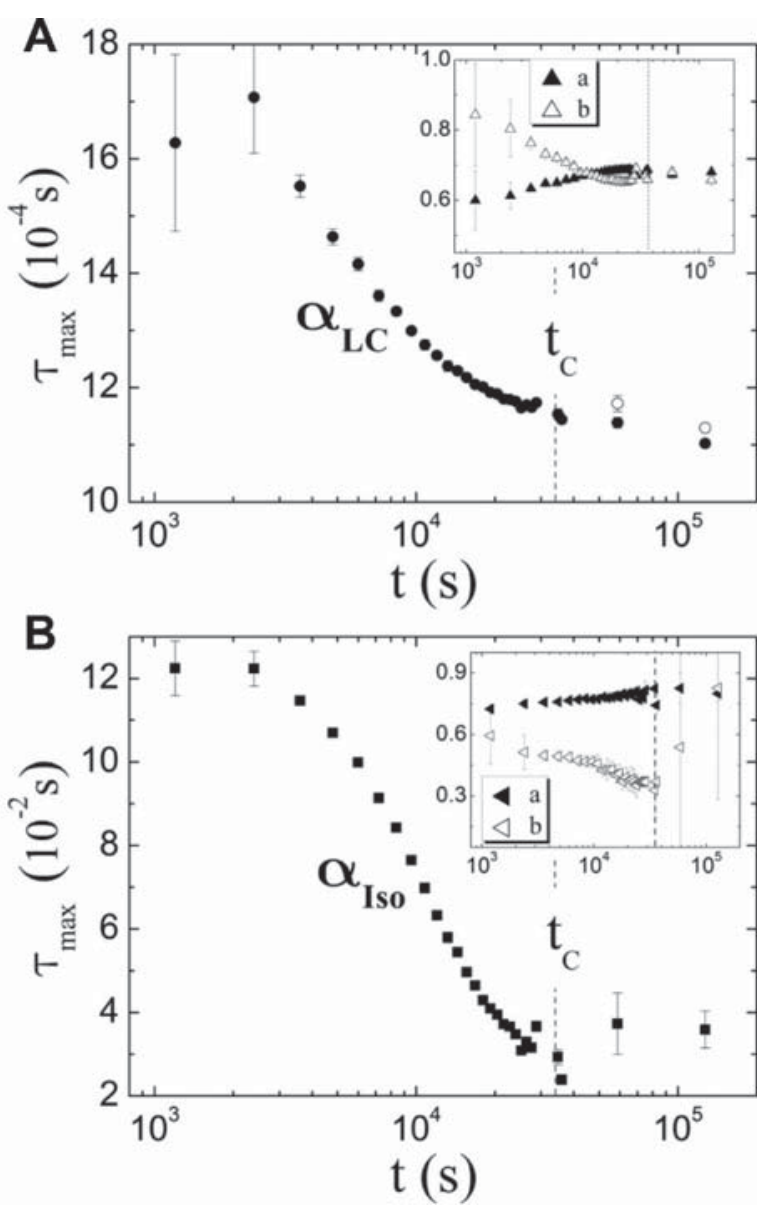

Figure 15. Temporal evolution of the relaxation time for the processes: (a) $\alpha_{\mathrm{LC}}$ and (b) $\alpha_{\text {iso }}$ during the mesophase formation at $30{ }^{\circ} \mathrm{C}$. The open symbols in panel a represent equivalent fittings when fixing $\Delta \varepsilon_{\text {iso }}=0$. The insets show the evolution of the HN shape exponents.

one), a similar sigmoidal trend is obtained (not shown). Fitting the long-time data with only one HN gives nearly the same $\tau_{\text {max,LC }}$ values (see the open symbols of Figure 15a). Finally, the shape of the exchanging $\alpha_{k}$ modes does not vary significantly during the phase transformation (see the insets of Figure 15).

In summary, the phase transition from the iso to the LC phase involves the substitution of the $\alpha_{\text {iso }}$ mode, associated to the segmental dynamic of the amorphous chains, by the faster $\alpha_{\mathrm{LC}}$ mode probably related to the motion of the elongated chains between smectic layers. The first stage of the kinetics, related to the smectic layer formation, practically takes the whole dielectric strength exchange between modes. This main process can be described by means of an Avrami kinetic model of exponent near to one, related to an athermal onedimensional crystallization scheme. In addition, an acceleration of the transient modes is observed during the phase transition.

3.2.3. Pressure Dependence of the $\alpha$ Relaxation. The influence of pressure on the $\alpha$ dynamics of iso and LC phases of PTEMeB has been also studied. The experiments have been carried out with the protocol described in section 2.2. A sample previously annealed for two days at $30{ }^{\circ} \mathrm{C}$, hence in the LC state, has been used for the two experiments performed below the isotropization point and also for the one below the mesophase SmA to SmC transition: $T_{\mathrm{sp}}=30^{\circ}, 38^{\circ} \mathrm{C}<T_{\text {SmC-A }}$ 
$<T_{\text {iso. }}$. The isobaric dielectric loss curves for $38^{\circ} \mathrm{C}$ are shown in Figure 16a and point out that the $\alpha_{\mathrm{LC}}$ mode shifts to higher
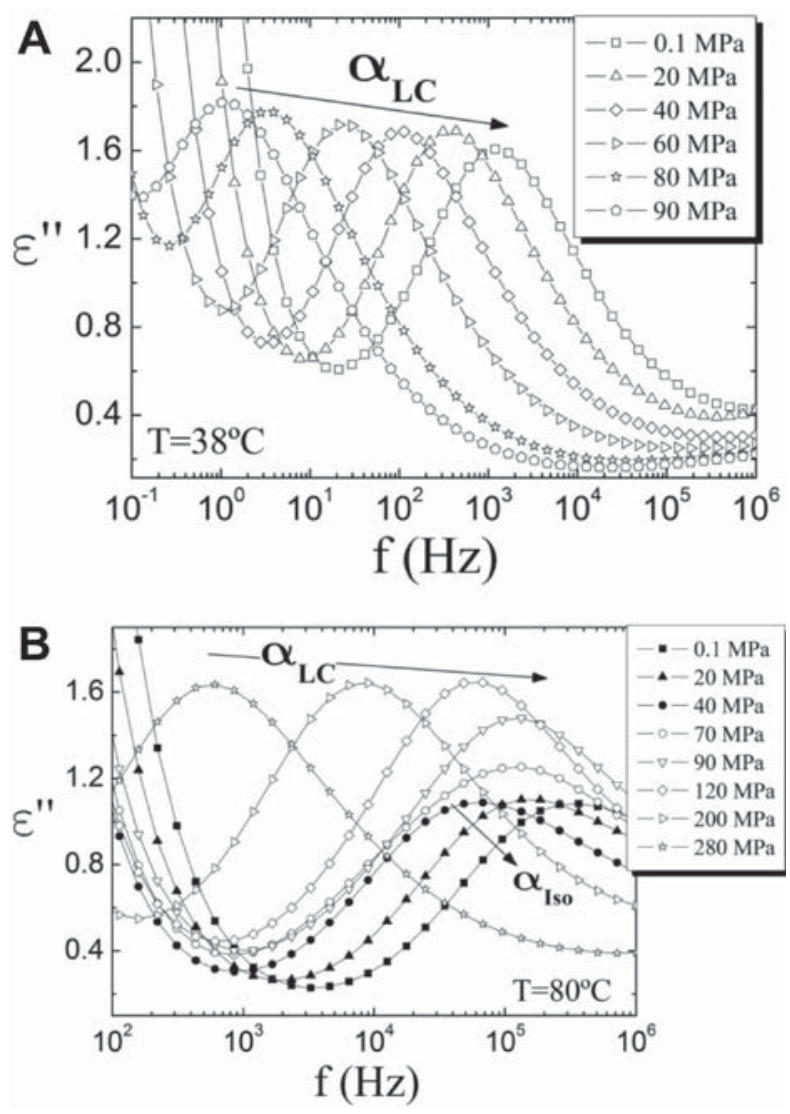

Figure 16. Isobaric dielectric loss curves at different pressures (given in the legend) for the temperatures: (a) $T=38^{\circ} \mathrm{C}<T_{\text {SmC-A }}<T_{\text {iso }}$ and (b) $T=80^{\circ} \mathrm{C}>T_{\text {iso. }}$ The $\alpha_{\mathrm{LC}}$ and $\alpha_{\text {iso }}$ processes are remarked.

frequencies for decreasing pressure (contrary to the effect of increasing temperature). The higher the pressure, the higher the density and the lower the free volume, therefore, the slower the dynamic glass transition. Comparing these results with the experiment at atmospheric pressure of Figure 11a one can observe differences on the peak intensity, showing higher values in the results of Figure 16a (aprox. $\Delta \varepsilon^{\prime \prime}{ }_{\text {peak }} \approx 0.4$ ). This discrepancy can be explained in terms of a higher parallel dipolar correlation in the present case, possibly due to a collective reorientation of the mesophase structure induced by the sudden increase of the pressure to $p_{\max }$ (somehow like a weak ferroelectric effect).

The rest of the temperatures studied $\left(60,70\right.$, and $\left.80^{\circ} \mathrm{C}\right)$ are higher than the isotropization temperature, and hence, the sample is initially in the iso state before applying pressure. For the sake of example, Figure 16b shows the results for $T_{\mathrm{sp}}=80$ ${ }^{\circ} \mathrm{C}$ increasing to the maximum working pressure, waiting for the sample stabilization (on $T$ and $p$ ) and recording the different isobars. It is observed that, when the pressure is decreased down to $90 \mathrm{MPa}$, the loss curves reverse their tendency by shifting to lower frequencies, and the intensity decreases and the width of the peaks increases. The slowing down trend continues up to the $40 \mathrm{MPa}$, whereas for the isobars at lower pressures, the opposite trend is found. This behavior is analogous to the one observed in the experiment of the Figure 11a, when both the SmC to SmA transition and the isotropization take place. The comparison of both experiments points out that, at high pressures (above $90 \mathrm{MPa}$ ), the sample is in a liquid crystal phase, whereas at low pressures (below 40 $\mathrm{MPa})$, the system becomes an isotropic liquid. The slowing down of the peaks observed in between can be tentatively assigned to a transition between mesophases of different nature as in the atmospheric pressure experiment. Taking into account the experimental protocol used, it can be stated that the sudden increase of pressure at the beginning of the experiment has induced a fast isotropic liquid to liquid crystal transition. It has to be remarked that, at $p_{\max } \approx 300 \mathrm{MPa}$, a slow mesophase formation, like the one of Figure 13 (at $p=0.1 \mathrm{MPa}$ ), has not been observed.

The dielectric loss curves of Figure 16 can be fitted by eq 1 using an HN function for the iso or the LC mode $\left(k=\alpha_{\text {iso }}\right.$ or $\alpha_{\mathrm{LC}}$ ). In the transition region (the slowing down region, e.g., 90 $\mathrm{MPa} \geq p \geq 40 \mathrm{MPa}$ for $T_{\mathrm{sp}}=80^{\circ} \mathrm{C}$ ), the peak is also described with only one broad $\mathrm{HN}$ function. This is contrary to the method followed in the equivalent transition region of the atmospheric pressure experiment where two contributions were used (see Figure 11a). In the present case, there are not structural studies justifying the deconvolution of the peak in two modes. Finally, the conductivity contribution and a $\beta$ low frequency wing (fixing the shape and relaxation time of the CC model) of eq 1 are also necessary for describing the data in the entire frequency range. The relaxation times of the $\mathrm{HN} \alpha$ modes are shown in the Arrhenius plot of Figure 17. For $T>$

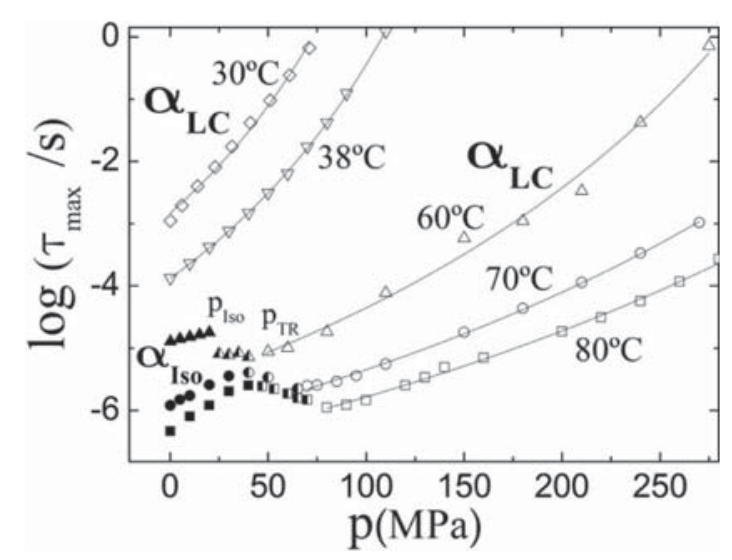

Figure 17. Pressure dependence of the relaxation time for the $\alpha_{\mathrm{LC}}$ (open symbols) and $\alpha_{\text {iso }}$ (closed) modes for different temperatures. The lines are fittings to eq 4 with the parameters given in Table 1 . The limits of transition region $\left(p_{\mathrm{TR}}, p_{\text {iso }}\right)$ are remarked for the $60{ }^{\circ} \mathrm{C}$ curve (half open symbols).

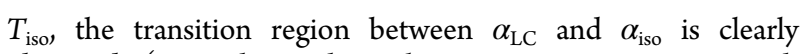
observed (see where the relaxation time increases with decreasing pressure). In order to describe the pressure dependency of the relaxation, a modification of the VFT, proposed by Johary and Walley, has been used ${ }^{40}$

$$
\tau_{\max }=\tau_{0} \exp \left(\frac{D_{\mathrm{p}} p}{p-p_{0}}\right)
$$

where $\tau_{0}$ is the relaxation time at $p=0$ (in practice at atmospheric pressure, $0.1 \mathrm{MPa}$, since the experimental error is 10 times higher, $\Delta p=1 \mathrm{MPa}$ ). The $D_{\mathrm{p}}$ parameter is constant for a wide range of pressures and temperatures, and $p_{0}$ is the 
pressure value at which the relaxation time diverges. ${ }^{41}$ The fitting curves can be seen in Figure 17, with parameter values given in Table 1 . The common value of $D_{\mathrm{p}}$ has been obtained

Table 1. Parameter Values of Fitting the Pressure Dependence of the Relaxation Times (See Figure 17) to eq 4

\begin{tabular}{cccc}
$T\left({ }^{\circ} \mathrm{C}\right)$ & $\log \tau_{0}( \pm 0.3)$ & $p_{0}( \pm 10 \mathrm{MPa})$ & $D_{\mathrm{p}}( \pm 0.5)$ \\
30 & -2.8 & 250 & 15.8 \\
38 & -3.9 & 300 & 15.8 \\
60 & -5.7 & 625 & 15.8 \\
70 & -6.3 & 830 & 15.8 \\
80 & -6.6 & 930 & 15.8 \\
\hline
\end{tabular}

from the free fitting of the best set of data, at $38{ }^{\circ} \mathrm{C}$, which has enough curvature to determine it accurately. The dielectric strength of the iso mode increases with pressure and is nearly constant for the LC mode. The transition is continuous, opposite to the $\varepsilon^{\prime \prime}$ peak jump observed in Figure 16b, given the broadening of the peaks in the iso phase (results not shown).

The pressure at which the glass transition of the liquid crystal takes place can be defined as $p_{\mathrm{g}}=p(\tau=100 \mathrm{~s})$ and calculated by the extrapolation of the $\tau_{\alpha, \mathrm{LC}}(p)$ dependence at the corresponding value of the relaxation time. However, the isotropization pressure, $p_{\text {iso, }}$ can be estimated as the end of the slowing down of the $\alpha$ mode (see Figure 17), similarly to the $p$ $=0.1 \mathrm{MPa}$ experiments of Figure 12. These two critical pressures added to the beginning of the transition region, namely, $p_{\mathrm{TR}}$ (see Figure 17), can be used to build a phase

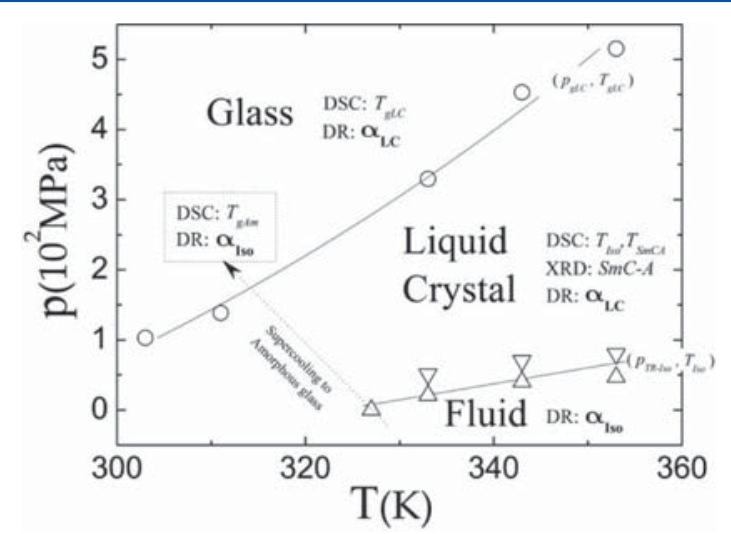

Figure 18. Phase diagram of the system and characteristic magnitudes and processes related to the different phases, derived from the different experimental techniques used (DSC, differential scanning calorimetry; XRD, X-ray diffraction; and DR, dielectric relaxation spectroscopy). The value of the glass transition pressures (circles) are obtained by extrapolation of the dependence of the relaxation times to $\tau=100 \mathrm{~s}$, and fitted to eq 5 . Whereas the transition region between the LC phase and the fluid ( $p_{\mathrm{TR}}-p_{\text {iso }}$ triangles) are extracted from the slowing down of the $\alpha$ process (the straight line is a guide for the eye).

diagram of the system, as shown in Figure 18. The strong glass transition dependence can be parametrized by the equation ${ }^{42,43}$

$$
T_{\mathrm{g}}(p)=T_{\mathrm{g}}(0)\left(1+\frac{b}{a} p\right)^{1 / b}
$$

where $a=1.7 \pm 0.5 \mathrm{GPa}, b=4.2 \pm 0.2$, and $T_{g}(0)=15 \pm 0.5$ ${ }^{\circ} \mathrm{C}$, the same as the calorimetric value (see section 3.1 ).
In summary, a fast phase transformation between isotropic phase and mesophase has been induced by a sudden pressure increase. Later on, the dynamic glass transition has been followed by stepwise decompression, and the isothermal isotropization process is revealed by the slowing down of the $\alpha$ relaxation as the pressure decreases. The relaxation time analysis allows to estimate the glass transition pressure dependence and to build a phase diagram for the PTEMeB liquid crystal.

\section{CONCLUSIONS}

The main chain liquid crystalline polymer, PTEMeB, was designed with the methyl group as a lateral substituent. This modification improved the stability of the isotropic liquid, i.e., the mesophase formation is relatively slow, and it has been possible to quench the system into the amorphous glass. The thermal transitions for both the liquid crystalline and amorphous glasses have been measured independently. The glass transition temperature of the mesophase turned out to be lower than the one of the amorphous state. This is consistent with the dynamical behavior: the $\alpha$ relaxation of the iso phase, supercooled or melted from the mesophase, is slower than the $\alpha$ relaxation of the LC state. It suggests that, in the liquid crystal glass, the molecular origin of the dynamic glass transition is other than the cooperative segmental motion of the chains, characteristic of amorphous polymers. Moreover, it has been proposed in the literature ${ }^{31}$ that the molecular mechanisms of the mesophase glass transition are the rotational and translational motions of the elongated chains that require less free volume.

The structural studies of the liquid crystal reveal the coexistence of two smectic phases at low temperatures ( $\mathrm{SmA}$ and $\mathrm{C}$ ). Additionally, the tilted $\mathrm{SmC}$ is transformed into the orthogonal $\mathrm{SmA}$ a few degrees below the isotropization. Taking this into account, the $\alpha$ relaxation peaks in the transition region have been analyzed in terms of the different transient phases.

The real-time phase transition from the isotropic liquid to the liquid crystal phase involves the $\alpha$ dynamics exchange. The first stage of the kinetics, related to the formation of the smectic planes, ${ }^{8}$ has been analyzed in terms of an Avrami kinetic model of exponent around unity. This can be understood as an athermal nucleation scenario followed by the unidimensional growing (needle-like crystals) of the active nuclei. During the main transformation, the transient modes that composed the deconvolution of the $\alpha$ relaxations became faster. Similarly, the isosbestic points of the dielectric loss spectra shifted to higher frequencies. These facts suggest a second order effect that is not completely understood at the moment. A very slow second stage that involves only a minor dielectric strength change is followed, and it is related to the improvement of the smectic order. $^{8}$

Finally, the high pressure dielectric study of PTEMeB revealed that the fast pressure increase induces a fast mesophase formation. It seems that, at high pressures, the methyl lateral substituent is not enough to inhibit a comparatively fast transformation into the liquid crystal. The temperature and pressure dynamical characterization allowed to draw a phase diagram of the PTEMeB system comprising the liquid crystalline glass, a kinetically arrested state, and the structural phases: isotropic liquid and liquid crystal. 


\section{AUTHOR INFORMATION}

\section{Corresponding Author}

*E-mail: encinar@quim.ucm.es (M.E.); aranmg@ictp.csic.es (A.M-G.).

\section{Present Address}

${ }^{\perp}$ Instituto de Microelectrónica de Madrid, CSIC, Isaac Newton 8 (PTM), Tres Cantos, 28760-Madrid, Spain.

\section{Author Contributions}

${ }$ These authors contributed equally to this work.

\section{Notes}

The authors declare no competing financial interest.

\section{ACKNOWLEDGMENTS}

We acknowledge the financial support of MICINN, Projects FIS2009-14008-C02-01 and MAT2010-19883, and EU for the Marie Curie MULTIFLOW ITN. The synchrotron work was also supported by MICINN through specific grants for the access to the CRG beamline BM16 of the ESRF. The inestimable help of all the beamline personnel is also gratefully acknowledged. M.E. is grateful to MICINN for a Ph.D. fellowship.

\section{REFERENCES}

(1) MacDonald, W. A. In Liquid Crystal Polymers: From Structures to Applications; Collyer, A. A., Ed.; Elsevier: New York, 1992; p 407.

(2) Donald, A. M.; Windle, A. H. Liquid Crystalline Polymers (Cambridge Solid State Sciences Series); Cambridge University Press: Cambridge, U.K., 1992.

(3) Greiner, A.; Schmidt, H. W. In Handbook of Liquid Crystals; Demus, D., Goodby, J., Gray, G. W., Spiess, H. W., Vill, V., Eds.; Wiley-VCH: Weinheim, Germany, 1998; Vol. 3.

(4) Brand, H. R.; Finkelmann, H. In Handbook of Liquid Crystals; Demus, D., Goodby, J., Gray, G. W., Spiess, H. W., Vill, V., Eds.; Wiley-VCH: Weinheim, Germany, 1998; Vol. 3.

(5) Wunderlich, B.; Grebowicz, J. Adv. Polym. Sci. 1984, 60/61, 1-59.

(6) García-Bernabé, A.; Díaz Calleja, R.; Sanchos, M. J.; del Campo, A.; Bello, A.; Pérez, E. Polymer 2004, 45, 1533-1543.

(7) Fernández-Blázquez, J. P.; Bello, A.; Pérez, E. Polymer 2005, 46, 10004-10010.

(8) Ezquerra, T. A.; Martínez-Gómez, A.; Alvarez, C.; Alonso, E.; Sanz, A.; García-Gutiérrez, M. C.; Bello, A.; Pérez, E.; Funari, S. S.; Dommach, M. J. Non-Cryst. Solids 2005, 351, 2768-2772.

(9) Heinrich, W.; Stoll, B. Colloid Polym. Sci. 1985, 263, 895-898.

(10) Moura-Ramos, J. J.; Williams, G. Polymer 1991, 32, 909-915.

(11) Mierzwa, M.; Floudas, G.; Wewerka, A. Phys. Rev. E 2001, 64, 031703.

(12) Zentel, R.; Strobl, G.; Ringsdorf, H. Macromolecules 1985, 18, 960-965.

(13) Vallerien, S. U.; Kremer, F.; Boeffel, C. Liq. Cryst. 1989, 4, 7986.

(14) Brás, A. R.; Dionisio, M.; Huth, H.; Schick, C.; Schönhals, A. Phys. Rev. E 2007, 75, 061708.

(15) Floudas, G.; Mierzwa, M.; Schönhals, A. Phys. Rev. E 2003, 67, 031705.

(16) Pérez, E.; Pereña, J. M.; Benavente, R.; Bello, A. In Handbook of Engineering Polymeric Materials; Cheremisinoff, N. P., Ed.; Marcel Dekker: New York, 1997; p 383.

(17) Watanabe, J.; Hayashi, M.; Nakata, Y.; Niori, T.; Tokita, M. Prog. Polym. Sci. 1997, 22, 1053-1087.

(18) Bello, P.; Bello, A.; Riande, E.; Heaton, N. J. Macromolecules 2001, 34, 181-186.

(19) Martínez-Gómez, A.; Bello, A.; Pérez, E. Macromolecules 2004, 37, 8634-8640.

(20) Fernández-Blázquez, J. P.; Bello, A.; Pérez, E. Macromol. Chem. Phys. 2007, 208, 2611-2620.
(21) Martínez-Gómez, A.; Bello, A.; Pérez, E. e-Polymers 2008, 69, 116.

(22) Floudas, G. In Broadband Dielectric Spectroscopy; Kremer, F., Schönhals, A., Eds.; Springer: Berlin, Germany, 2003; p 295.

(23) Meyer, R. B.; Liebert, L.; Strzelezcki, L.; Keller, P. J. Phys. Paris Lett. 1975, 36, 69.

(24) Kremer, F.; Vallerien, S. U.; Zentel, R. Adv. Mater. 1990, 2, $145-147$.

(25) Mark, J. E., Ed., Polymer Data Handbook; Oxford University Press: New York, 1999.

(26) Martínez-Gómez, A.; Pérez, E.; Bello, A. Colloid Polym. Sci. 2010, 288, 859-867.

(27) Havriliak, S., Jr.; Havriliak, S. J. Dielectric and Mechanical Relaxation in Materials: Analysis, Interpretation, and Application to Polymer; Hanser Verlag: New York, 1997; Chapter 1.

(28) Cole, K. S.; Cole, R. H. J. Chem. Phys. 1941, 9, 341-351.

(29) Encinar, M.; Prolongo, M. G.; Rubio, R. G.; Ortega, F.; Ahmadi, A.; Freire, J. Eur. Phys. J. E 2011, 34, 1-14.

(30) Angell, C. A. Science 1995, 267, 1924-1935.

(31) Ahumada, O.; Ezquerra, T. A.; Nogales, A.; Balta-Calleja, F. J.; Zachmann, H. G. Macromolecules 1996, 29, 5002-5009.

(32) Sics, I.; Nogales, A.; Ezquerra, T. A.; Denchev, Z.; Baltá-Calleja, F. J.; Meyer, A.; Döhrmann, R. Rev. Sci. Instrum. 2000, 71, 1733-1736.

(33) Soccio, M.; Nogales, A.; Lotti, N.; Munari, A.; Ezquerra, T. A. Polymer 2007, 48, 4742-4750.

(34) Frohlich, H. Theory of Dielectrics: Dielectric Constant and Dielectric Loss; Clarendon Press: Oxford, U.K., 1968.

(35) Avrami, M. J. Chem. Phys. 1939, 7, 1103.

(36) Avrami, M. J. Chem. Phys. 1940, 8, 212.

(37) Menczel, J. D.; Prime, R. B. Thermal Analysis of Polymers, Fundamental and Applications; Wiley: New York, 2009.

(38) Haase, N.; Grigoriadis, C.; Butt, H. J.; Müllen, K.; Floudas, G. J Phys. Chem. B 2011, 115, 5807-5814.

(39) Grigoriadis, C.; Haase, N.; Butt, H. J.; Müllen, K.; Floudas, G. Soft Matter 2011, 7, 4680-4689.

(40) Johari, G. P.; Whalley, E. Faraday Symp. Chem. Soc. 1972, 6, 2341.

(41) Roland, C. M.; Hensel-Bielowka, S.; Paluch, M.; Casalini, R. Rep. Prog. Phys. 2005, 68, 1405-1478.

(42) Floudas, G. Prog. Polym. Sci. 2004, 29, 1143-1171.

(43) Andersson, S. P.; Andersson, O. Macromolecules 1998, 31, 2999-3006. 